\title{
Evidence for a chemical enrichment coupling of globular clusters and field stars in the Fornax dSph ${ }^{\star}$
}

\author{
Benjamin Hendricks ${ }^{1}$, Corrado Boeche ${ }^{2}$, Christian I. Johnson ${ }^{3}$, Matthias J. Frank ${ }^{1}$, Andreas Koch ${ }^{1}$, \\ Mario Mateo ${ }^{4}$, and John I. Bailey III $^{4}$
}

\footnotetext{
1 Zentrum für Astronomie der Universität Heidelberg, Landessternwarte, Königstuhl 12, 69117 Heidelberg, Germany e-mail: ben.hendricks@lsw.uni-heidelberg.de

2 Astronomisches Rechen-Institut, Zentrum für Astronomie der Universität Heidelberg, Mönchhofstr. 12-14, 69120 Heidelberg, Germany

3 Harvard-Smithsonian Center for Astrophysics, 60 Garden Street, MS-15, Cambridge, MA 02138, USA

4 Department of Astronomy, University of Michigan, 1085 South University, Ann Arbor, MI 48109, USA
}

Received 19 July 2015 / Accepted 2 October 2015

\begin{abstract}
The globular cluster H4, located in the center of the Fornax dwarf spheroidal galaxy, is crucial for understanding the formation and chemical evolution of star clusters in low-mass galactic environments. $\mathrm{H} 4$ is peculiar because the cluster is significantly more metalrich than the galaxy's other clusters, is located near the galaxy center, and may also be the youngest cluster in the galaxy. In this study, we present detailed chemical abundances derived from high-resolution $(R \sim 28000)$ spectroscopy of an isolated $\mathrm{H} 4$ member star for comparison with a sample of 22 nearby Fornax field stars. We find the $\mathrm{H} 4$ member to be depleted in the alpha-elements $\mathrm{Si}$, Ca, and Ti with $[\mathrm{Si} / \mathrm{Fe}]=-0.35 \pm 0.34,[\mathrm{Ca} / \mathrm{Fe}]=+0.05 \pm 0.08$, and $[\mathrm{Ti} / \mathrm{Fe}]=-0.27 \pm 0.23$, resulting in an average $[\alpha / \mathrm{Fe}]=-0.19 \pm 0.14$. If this result is representative of the average cluster properties, $\mathrm{H} 4$ is the only known system with a low $[\alpha / \mathrm{Fe}]$ ratio and a moderately low metallicity embedded in an intact birth environment. For the field stars we find a clear sequence, seen as an early depletion in $[\alpha / \mathrm{Fe}]$ at low metallicities, in good agreement with previous measurements. $\mathrm{H} 4$ falls on top of the observed field star $[\alpha / \mathrm{Fe}]$ sequence and clearly disagrees with the properties of Milky Way halo stars. We therefore conclude that within a galaxy, the chemical enrichment of globular clusters may be closely linked to the enrichment pattern of the field star population. The low $[\alpha / \mathrm{Fe}] \mathrm{ratios}$ of $\mathrm{H} 4$ and similar metallicity field stars in Fornax give evidence that slow chemical enrichment environments, such as dwarf galaxies, may be the original hosts of alpha-depleted clusters in the halos of the Milky Way and M31.
\end{abstract}

Key words. galaxies: clusters: individual: Fornax - globular clusters: individual: H4 - galaxies: abundances - galaxies: evolution galaxies: dwarf - stars: abundances

\section{Introduction}

Globular clusters (GCs) are an intriguing class of stellar systems and have been objects of interest for many decades. When resolved, they offer a unique possibility of studying their effectively coeval and mono-metallic stellar populations in detail. In distant galaxies, unresolved globular clusters serve as luminous beacons that can still be analyzed when individual field stars are too faint to observe (e.g., Brodie \& Strader 2006).

While the chemical (self-)enrichment in GCs after their formation has been studied in great detail in the recent past (see, e.g., Gratton et al. 2012, and references therein), relatively little is known about the chemical enrichment of these systems before their formation, that is, the chemical evolution of the proto-GC gas embedded in a galactic environment. Specifically, it is not clear whether GCs - despite their undoubtedly unique formation mechanism - reflect the chemical properties of the field stars in the host environment they are born in or whether they instead display distinct chemical enrichment properties.

The alpha-elements $(\mathrm{O}, \mathrm{Mg}, \mathrm{Si}, \mathrm{Ca}$, and $\mathrm{Ti})$ are an important instrument for studying the details of chemical enrichment

^ This article includes data gathered with the $6.5 \mathrm{~m}$ Magellan Telescopes located at Las Campanas Observatory, Chile. in stellar systems. These elements are synthesized almost exclusively in massive stars and therefore trace the contribution ratio between high-mass Supernovae II (SNe II; yielding high $[\alpha / \mathrm{Fe}]$ ) and low-mass Supernova Ia (SNe Ia; yielding low $[\alpha / \mathrm{Fe}]$ ) to the interstellar medium (ISM). As a result, the $[\alpha / \mathrm{Fe}]$ ratio is enhanced for the first, metal-deficient stars and subsequently drops with increasing $[\mathrm{Fe} / \mathrm{H}]$ as soon as $\mathrm{SNe}$ Ia start to contribute significantly to the enrichment. The increasing contributions from SNe Ia are seen as an inflection point or knee in plots of $[\alpha / \mathrm{Fe}]$ versus $[\mathrm{Fe} / \mathrm{H}]$. Consequently, the evolution of $[\alpha / \mathrm{Fe}]$ reveals the extent to which the galaxy can enrich its ISM before SNe Ia set in, and hence is a measurement for the chemical enrichment efficiency of the system (Tinsley 1979; Matteucci \& Brocato 1990; Lanfranchi \& Matteucci 2003). Since all stars are effectively coeval in a GC, their $\alpha / \mathrm{Fe}$-ratio represents the chemical enrichment status of the proto-cluster material at the time of formation.

Globular clusters in the Milky Way (MW) halo are found to be almost exclusively alpha-enhanced over the entire observed range of metallicities (Pritzl et al. 2005), indicating a fast chemical enrichment of the material from which they formed. Simultaneously, they fall on top of the enhanced $[\alpha / \mathrm{Fe}]$ plateau displayed by the field stars (e.g., Venn et al. 2004), and therefore no clear distinction between a uniform enrichment scenario and 


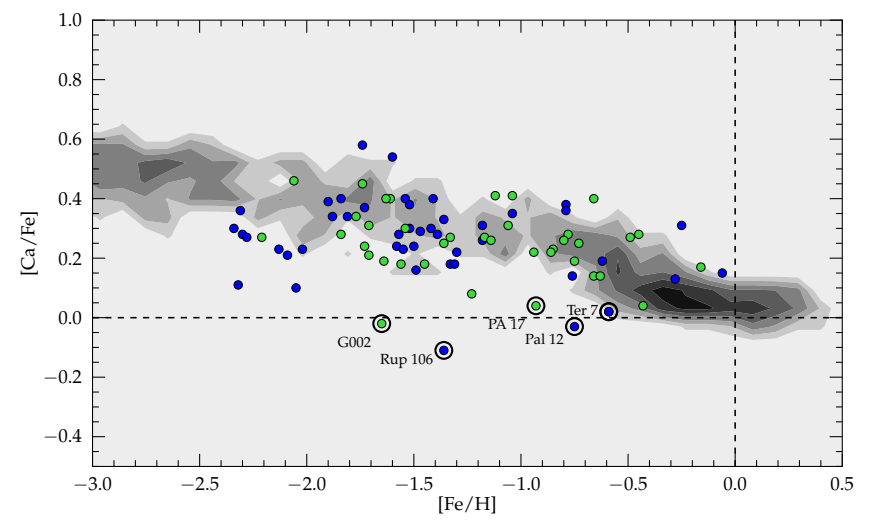

Fig. 1. Literature compilation of the chemical abundance pattern of $\mathrm{Ca}$ as a function of $[\mathrm{Fe} / \mathrm{H}]$. Symbols show GCs in the MW from Pritzl et al. (2005; blue) and M31 (green) from Colucci et al. (2014) and Sakari et al. (2015). The pattern of MW disc and halo stars are shown as a logarithmically-scaled number density distribution of arbitrary units (data from Venn et al. 2004 and Roederer et al. 2014). Outliers with significantly lower $[\mathrm{Ca} / \mathrm{Fe}]$ are highlighted with large open black circles.

a coupling with the field star properties can be made (see Fig. 1). A similar picture is found for the GC system in Andromeda (M31) where a large number of clusters have been studied recently by Colucci et al. (2014) and Sakari et al. (2015) using integrated-light spectroscopy. Although the alpha-evolution of field stars in M31 is not known given the extremely faint magnitudes of individual stars, the $[\alpha / \mathrm{Fe}]$ values of the GCs agree well with the field stars and the GCs of the MW. Notably, the clusters on the high-metallicity end in the sample of Colucci et al. (2014) possibly show lower alpha-abundance ratios and may follow the knee of MW field stars, which could be a hint for a common chemical enrichment pattern between field stars and clusters.

Both galaxies host a few, but interesting, outliers with significantly lower $[\alpha / \mathrm{Fe}]$ ratios with respect to the field stars at comparable metallicity: Terzan 7, Palomar 12, and the younger system Ruprecht 106 in the MW (Sbordone et al. 2005; Cohen 2004; Brown et al. 1997), G002 and PA17 in M31 (Colucci et al. 2014; Sakari et al. 2015). While Terzan 7 and Palomar 12 are commonly associated with the currently disrupting Sagittarius dwarf galaxy (see Law \& Majewski 2010, and references therein), the other clusters are orphans without a known parental system. Only because of their abnormal chemical signatures is it speculated that they have formed in smaller satellite systems with slower chemical enrichment, and have subsequently been stripped from their hosts during the accretion (e.g., Villanova et al. 2013; Colucci et al. 2014). In this scenario, the GCs need to share the fingerprint of slow chemical enrichment inherent to the host galaxy. Furthermore, it would be possible to generally use clusters with peculiar alpha-abundances for chemical tagging of accreted systems.

The only Local Group galaxy known to host GCs while its stars are simultaneously alpha-depleted at a metallicity of Ruprecht 106 or G002 is the Fornax dwarf spheroidal. This galaxy therefore may provide a unique test case to address the key questions of whether GCs share the chemical enrichment pattern of their host galaxy and, as a consequence, whether it is possible to use peculiar alpha-abundances (i.e., those deviating from the field stars) for chemical tagging of accreted systems.

Fornax is considered a "classical" dwarf spheroidal galaxy. It is one of the most massive Galactic satellites with a few $10^{7} M_{\odot}$ (McConnachie 2012), an extended star formation history

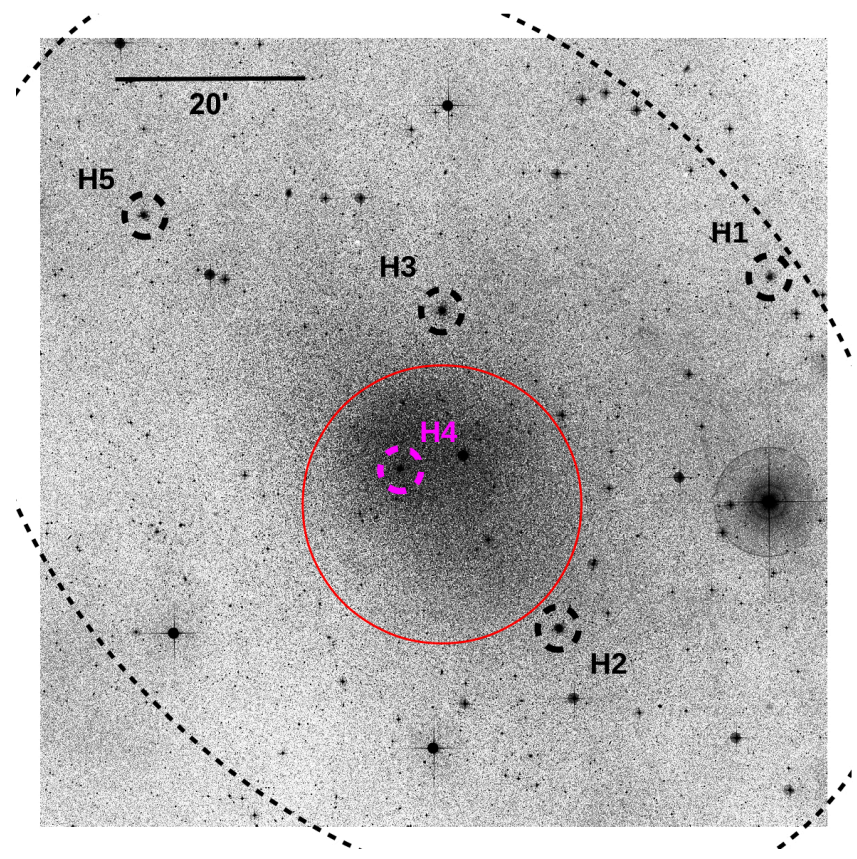

Fig. 2. DSS image of the Fornax dwarf spheroidal galaxy. The position of the five GCs are highlighted. H4 is located close to the center of the galaxy and therefore suffers from strong contamination of the surrounding field. The red circle shows the approximate pointing and field-of-view of $M 2 F S$ with a radius of $14.65^{\prime}$. The dashed ellipse indicates the tidal radius of the galaxy.

(de Boer et al. 2012b; del Pino et al. 2013), and consequently hosts stars of a broad range of metallicities (Battaglia et al. 2006; Hendricks et al. 2014b). Moreover, the galaxy hosts its own population of five GCs (see Fig. 2). Four of the Fornax GCs are metal-poor with $[\mathrm{Fe} / \mathrm{H}] \leq-2.0$ (Letarte et al. 2010), old (Buonanno et al. 1998) and alpha-enhanced (Letarte et al. 2010; Larsen et al. 2012), and by that resemble typical MW halo clusters. The remaining cluster (named H4, following Hodge 1961) is an outlier in many respects: it is significantly more metalrich, around $[\mathrm{Fe} / \mathrm{H}]=-1.4$ (Strader et al. 2003; Larsen et al. 2012), and possibly younger than the other clusters (Buonanno et al. 1999). Most importantly, it has only recently been shown that field stars in Fornax become alpha-depleted at very low metallicities with a knee in the alpha-evolution most likely below $[\mathrm{Fe} / \mathrm{H}] \approx-1.9$ dex (Hendricks et al. 2014a; Lemasle et al. 2014). As a consequence, $\mathrm{H} 4$ is located at a metallicity where the field stars in Fornax are clearly alpha-depleted. This contrasts with the composition characteristics of similar metallicity MW field stars, which are alpha-enhanced. Therefore, H4 provides a unique opportunity to test if GCs share the chemical enrichment pattern of their host galaxy, and if it is possible to use peculiar alpha-abundances to chemically identify accreted systems such as Ruprecht 106 in the MW or G002 in M31.

Despite its importance for understanding GC formation and evolution in Fornax, $\mathrm{H} 4$ is the only cluster in the galaxy for which no individual member stars have been analyzed to date. $\mathrm{H} 4$ is located very close to the center of the galaxy and is therefore severely contaminated by field stars. Additionally, it happens to be the most compact of all Fornax GCs, and at a distance of $\sim 147 \mathrm{kpc}$ its core radius amounts to only 2.64" (Mackey \& Gilmore 2003). From ground-based telescopes, H4 is only resolved into individual stars in its outer regions where the fraction of cluster member and field star contaminants is about equal. 
In the study presented here, three likely individual member stars of the cluster $\mathrm{H} 4$ have been identified. For one of them, we derived detailed chemical abundances and put them in direct comparison with field stars of both the Fornax dwarf spheroidal and the MW at similar metallicity. The goal of this work is to shed light on the question of whether or not the chemical enrichment history of field stars within a galaxy is imprinted on its GC system.

The paper is organized as follows. In Sect. 2, we summarize our target selection, observing setup, and data reduction. Section 3 gives details about the chemical analysis, which is subsequently presented in Sect. 4 together with an age analysis of H4. In Sect. 5, we discuss the impact of our findings with respect to the nature of alpha-depleted GCs, the nature of H4 itself, and the chemical enrichment properties of Fornax. Finally, in Sect. 6, we summarize our main results.

\section{Data acquisition and reduction}

Finding individual and bright member stars in $\mathrm{H} 4$ is a challenging endeavor. A multi-object spectrograph is necessary in order to efficiently observe cluster members. Furthermore, the cluster's large distance with accessible targets located at 10-60" from the cluster center, means that the instrument must be capable of densely packing many objects onto a small spatial scale. To avoid contamination, the apertures also need to be small and able to be placed on the field-of-view with high precision. Finally, with most targets fainter than $V=19.0 \mathrm{mag}$, exposure times to obtain sufficient signal-to-noise for a chemical analysis are long and suffer from accumulating cosmic rays.

\subsection{Observations, instrument, and setup}

For this project, we used the Michigan/Magellan Fiber System (M2FS, Mateo et al. 2012) and MSpec spectrograph, a fiberfed spectrograph mounted on the Nasmyth-east port of the Magellan-Clay $6.5 \mathrm{~m}$ telescope at Las Campanas Observatory. For $M 2 F S$, we used the Bulge_GC1 setup and $125 \mu \mathrm{m}$ slit (see Johnson et al. 2015), which allows a simultaneous observation of up to 48 targets at a resolving power of $R \approx 28000$ and a continuous wavelength coverage from 6140 to $6720 \AA$. The observations have been carried out during three consecutive nights in December 2014. With 8 individual exposures, a total of $6.7 \mathrm{~h}$ have been observed on target with typical seeing conditions around $0.8^{\prime \prime}$.

\subsection{Target selection}

Due to the central location of $\mathrm{H} 4$ in the field of the Fornax dwarf galaxy, the cluster is heavily contaminated by Fornax field stars. The contamination fraction in the outer half of the cluster tidal radius is higher than $50 \%$, and still amounts to $\geq 20 \%$ around the cluster center (see Sect. 4.1). A careful target selection is critical to maximize the fraction of actual $\mathrm{H} 4$ members, while simultaneously avoiding blended stars in the heavily crowded area. In addition to bona-fide $\mathrm{H} 4$ cluster members, we also deploy some of the fibers on bright field stars in the vicinity of the cluster to allow a direct comparison between cluster and galactic properties.

\subsubsection{GC candidate member stars}

For our target selection, we used archival Hubble Space Telescope imaging taken with the Wide-Field Planetary Camera

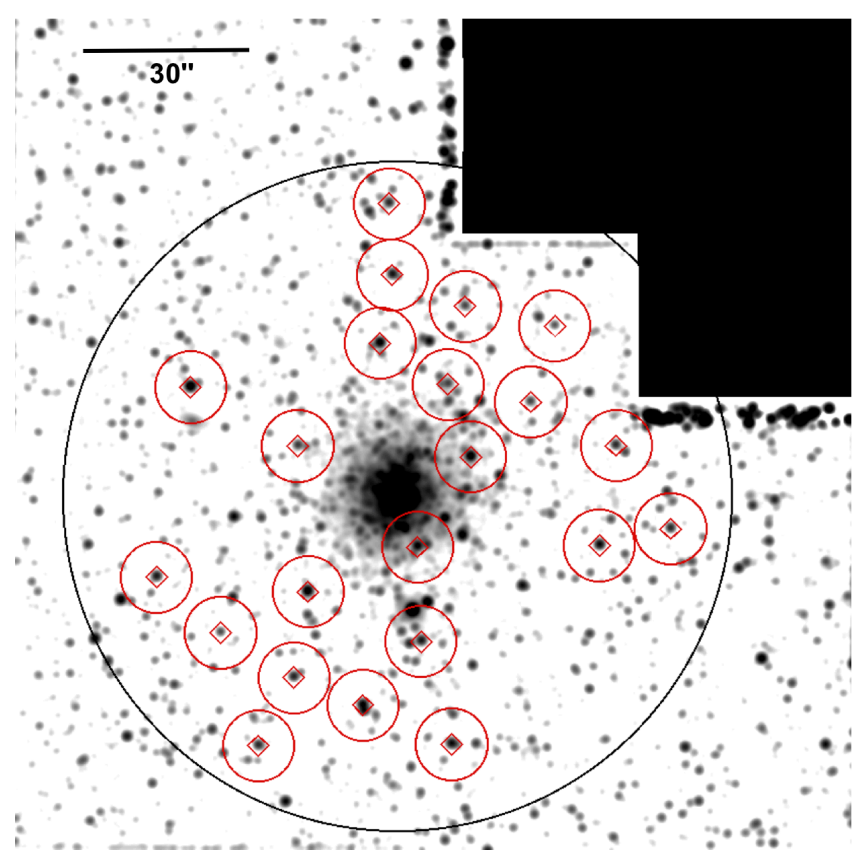

Fig. 3. Location of our targets in the GC H4, shown on the WFPC2 $F 555 \mathrm{~W}$-band mosaic and artificially degraded to a ground-based seeing of $1^{\prime \prime}$. The tidal radius of the cluster $\left(r_{t}=1^{\prime}\right)$ is shown in black and our targets are highlighted with red symbols. Red boxes are $2^{\prime \prime} \times 2^{\prime \prime}$ and mimic the actual fibersize while the circles are $6.5^{\prime \prime}$ in radius and visualize the minimum allowed spacing between individual fibers for M2FS.

2 (WFPC2) in programme 5637 (PI: Westphal). The data consist of two deep $(1100 \mathrm{~s})$ and one shallow $(60 \mathrm{~s})$ exposure in both of the $F 555 W(V)$ and $F 814 W(I)$ bands, and were first published by Buonanno et al. (1999). We retrieved the pipeline-reduced individual images from the STSCI archive and performed pointspread function fitting photometry using HSTPHOT (version 1.1; Dolphin 2000), following the same procedure described in more detail in Frank et al. (2012). Briefly, residual shifts of the images were determined by initially running HSTPHOT on each image separately and cross-matching the resulting catalogs. After the creation of cosmic-ray masks, the well-aligned deep exposures in each filter were co-added, and the $F 555 \mathrm{~W}$ deep co-added image was used as a detection image in the simultaneous photometry from all frames.

The output of HSTPHOT provides charge-transfer-efficiencyand aperture-corrected magnitudes in the HST system $(F 555 W$ and $F 814 W$ ), as well as magnitudes transformed to the JohnsonCousins $V$ and $I$ bands, based on the updated calibration and photometric transformation coefficients of Dolphin (2009). In the following, we use these $V$ and $I$ band magnitudes.

For astrometry, we created a mosaic of all $F 555 \mathrm{~W}$-band exposures (see Fig. 3) using MULTIDRIZZLE (Koekemoer et al. 2006), and transformed the photometric catalogs of the camera's four individual chips to this reference image, in order to correct for geometric distortion. We corrected for shifts in absolute RA and Dec by matching our catalog to the 2MASS astrometric system (Cutri et al. 2003), using the VIKING survey DR1 source catalog (Edge et al. 2013) as an intermediate step in the crossmatch because the 2 MASS point source catalog is very sparse in the $\sim 2.6^{\prime} \times 2.6^{\prime}$ field-of-view of the WFPC2 pointing.

Possible targets are selected from the red giant branch (RGB) and comprise stars from the RGB-tip down to magnitudes as faint as $V=20.5 \mathrm{mag}$. Although the RGB is fairly broad with a 


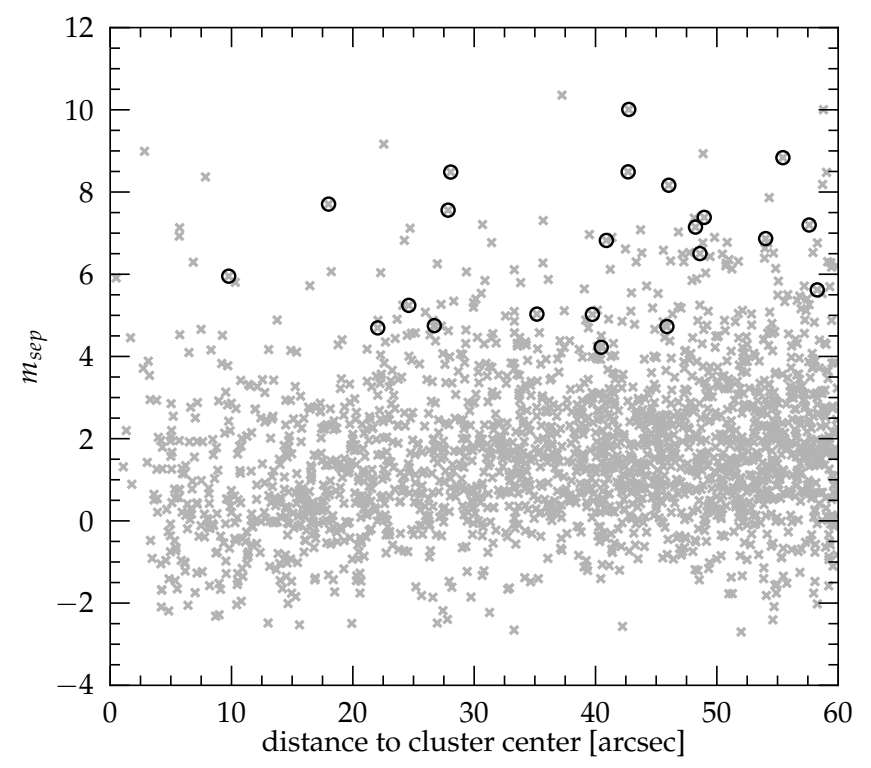

Fig. 4. Plot of the separation index defined in the text for stars in the GC H4 as a function of central distance. Gray crosses denote all red giant stars projected on the sky near the cluster, and our targets are highlighted with black circles. While $m_{\text {sep }}=0.0$ means that half of the star light comes from blending neighbors, $m_{\text {sep }}=4.0$ and 5.0 means $\sim 2.5 \%$ and $1.0 \%$ contamination, respectively.

possible color split for the brightest stars, it is not clear whether this is a signature of the GC population on top of a field stars mix or whether it simply reflects the intrinsic spread in the field star population. Therefore, we did not further constrain our selection to a specific part of the RGB, as for example done in Carretta et al. (2010a) for the case of M54 in the center of the Sagittarius dwarf spheroidal, where a cleaner separation between populations could be made. We also refrain from placing an individual fiber at the (unresolved) center of the cluster. The analysis of the resulting integrated-light spectrum would be inferior to classical drift-scan methods because the integrated spectrum does not consist of an entire population (which can be modelled at varying levels of accuracy) but may contain only some dozens of stars of essentially unknown origin and parameters.

Blending is a serious problem for stars in $\mathrm{H} 4$, specifically in the inner regions of the cluster where membership likelihood is highest, and we have to take into account the additional seeing from ground-based observatories in contrast to the HST images. To quantify the amount of blending and to exclude significantly flux-contaminated stars in advance, we calculate a separation index, developed by Stetson et al. (2003). In detail, we use the magnitude of each star in the HST catalog to calculate its flux, which we subsequently smooth with a Gaussian profile according to the seeing of our observations. We then calculate the flux ratio between the target star and all neighbors in the environment at the star's central position and express the result as a magnitude $\left(m_{\text {sep }}\right)$. Finally, we exclude all stars from our target list with a flux contamination higher than $5 \%$, equivalent to $m_{\text {sep }} \geq 3.25$. Our final targets typically have $m_{\text {sep }} \geq 5.0$, and by that suffer less than $1 \%$ contamination from neighboring stars (see Fig. 4).

\subsubsection{Field stars}

Field star targets of the Fornax dwarf spheroidal were selected from the catalog of Battaglia et al. (2008), which provides calcium triplet metallicities for nearly 1000 bright RGB stars that are cleaned from most of the contaminating Galactic halo foreground by velocity cuts. From this catalog, we purposefully picked rare, metal-poor stars with $[\mathrm{Fe} / \mathrm{H}] \leq-1.4$ dex to trace the alpha-element evolution at the metallicity of $\mathrm{H} 4$ and at the expected position of the $[\alpha / \mathrm{Fe}]$ knee. The selected field stars belong to the upper part of the RGB and consequently have similar magnitudes to the $\mathrm{H} 4$ candidate stars. Since they are located close to the cluster itself, our field star targets are all located in the central part of Fornax $\left(r \leq 0.3^{\circ}\right)$ and hence within the core radius of the galaxy.

\subsection{Data reduction}

The general spectroscopic data reduction process follows the description in Johnson et al. (2015). Using IRAF routines, the individual amplifier images on each CCD were separately trimmed, bias corrected and subsequently rotated, translated, and combined into one exposure per CCD. Next, we used the IRAF task DOHYDRA to extract the individual orders of each spectrum. This task has been written to extract spectra taken with the WIYN and Blanco Hydra spectrographs, and it can be applied to most multifiber echelle datasets. The routine includes aperture identification and tracing, scattered light removal, flat-field correction, throughput- and wavelength-calibration, and a basic cosmic-ray removal. Sky-subtraction within DOHYDRA is skipped and performed later using a master-sky frame from the combined sky fibers of both CCDs.

It is important to emphasize that DOHYDRA is called separately on each of the six orders for individual spectra. As a consequence, the extracted parts of any full spectrum underwent an individual data reduction process and therefore possibly display different systematic signatures imprinted by the individual extraction steps. This allows us to verify the robustness of our analysis against such possible systematics by comparing the results from different orders (see Sect. 3.2.3).

After sky subtraction, the individual exposures have been summed using a weighted average based on the typical S/N of the brightest targets in each frame, to maximize combined signal-to-noise. The heliocentric velocity was removed from each frame prior to co-addition in order to account for differences of up to $0.7 \mathrm{~km} \mathrm{~s}^{-1}$ between individual frames. Finally, the spectra are continuum normalized and the full wavelength range is recovered by combining the individual orders.

Compared to the data in Johnson et al. (2015), our targets are extremely faint with long individual exposure times and low signal-to-noise ( $\leq 10$ per pixel) within the individual exposures. This introduces some complications in the data reduction and requires some additional steps. First, given the long individual exposure times of up to $1 \mathrm{~h}$, the images suffer from severe cosmicray contamination for which DOHYDRA cannot properly account. The multitude of cosmic-ray features not only possibly spoil absorption features in the spectra, but also may pose a problem for fiber tracing, throughput calibration, and later to sky subtraction and continuum placement. For this reason, we use the Python implementation of LACOSMIC ${ }^{1}$ (van Dokkum 2001) on our $2 \mathrm{~d}$ images prior to the DOHYDRA task, which yields a significant improvement on our results. However, we cannot be sure if absorption features, initially affected by cosmic-rays, are recovered to their intrinsic, unbiased properties. Therefore, we generate a cosmic-ray mask in order to flag any regions in a spectrum which have been possibly biased by cosmic-ray removal.

\footnotetext{
1 Available online at http://obswww.unige.ch/ tewes/ cosmics_dot_py
} 
Unfortunately, DOHYDRA does not propagate bad pixel masks through the reduction. We therefore perform the extraction process twice, once on the images with and once on the images without previous correction by LACOSMIC. Then, we flag all wavelength regions where we observe a significant $(\geq 5$ sigma of continuum noise) difference between the extracted versions. Finally, we combine the regions from all individual exposures to build the cosmic-ray mask. Later, we will use this mask to test the robustness of our abundances by excluding these regions during the analysis (see Sect. 3.2.3).

Despite the initial throughput calibration, we observe flux variations between individual fibers, including science and sky fibers. This necessitates a sophisticated sky-subtraction for which we use the SKYTWEAK task in IRAF. The SKYTWEAK task allows for wavelength shifts and rescaling of the flux in the master-sky spectrum to minimize residuals of the most prominent emission features. The master-sky is generated by the average of 5 sky fibers distributed among both CCDs, and includes a min-max rejection algorithm to clear the spectrum of any remaining cosmic-rays or other contamination. In low signal-tonoise spectra with flux only $\sim 10$ times above the sky level, sky residuals will have a non-negligible impact on any chemical analysis. Similar to the cosmic-ray mask, we therefore generate a sky-mask where we flag wavelength regions with initially strong sky emission lines and test the sensitivity of our chemical analysis to these regions. The reddest order in our setup (order 57; 6653-6720 A) does not contain any significant sky emission features that can be used for rescaling. As a consequence, the level of sky subtraction is less reliable than for the other orders, and we therefore exclude this region from the chemical analysis for all stars.

Finally, some orders of some spectra are affected by an internal Littrow ghost reflection (e.g., Burgh et al. 2007; see also Johnson et al. 2015, their Fig. 1). Such regions are clearly visible as a strongly deviating continuum flux, and we exclude these regions from the forthcoming analysis.

After data reduction, we obtain a total of 40 science spectra (18 field stars and 22 stars within the tidal radius of $\mathrm{H} 4$ ). The signal-to-noise distribution, measured at $\sim 6388 \AA$, ranges from $\sim 40$ per pixel for the brightest targets to $\leq 10$ for the faintest objects. We estimate the final resolution of our spectra from the width of several clean sky emission lines and find a constant $R=\lambda / \Delta \lambda \approx 28000$ over the full wavelength range.

\section{Data analysis}

\subsection{Radial velocities}

We determine radial velocities for each star using the IRAF task FXCOR and a template spectrum convolved to the resolution of our observations. As template we used a synthetic spectrum ${ }^{2}$ with $T_{\text {eff }}=4250 \mathrm{~K}, \log g=1.0$, and $[\mathrm{Fe} / \mathrm{H}]=-1.4$, which is close to what we expect for $\mathrm{H} 4$ cluster members and suitable for our complete sample.

To obtain the most precise velocity determination, we use

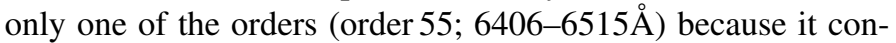
tains a series of deep, unblended absorption lines, it is relatively free of strong sky emission, and it does not contain broad lines nor strong telluric bands. Where applicable, we test the outcome with other orders and find consistent results within the uncertainties.

\footnotetext{
2 Obtained from the Coelho et al. (2005) library of high-resolution synthetic stellar spectra.
}

Velocity uncertainties reported by FXCOR are typically $\leq 1.0 \mathrm{~km} \mathrm{~s}^{-1}$ (between 0.2 and $2 \mathrm{~km} \mathrm{~s}^{-1}$, depending on $\mathrm{S} / \mathrm{N}$ ). We additionally estimate the uncertainty from the standard deviation of the individual exposures for each star, which yield very similar results to the FXCOR values. Although less critical than for the chemical analysis, we want to make sure that our velocities are not strongly biased by residual artifacts from sky emission or cosmic rays. Therefore, we determine velocities once using the full wavelength range of order 55 and once without the masked regions in the sky- and cosmic-ray mask. Reassuringly, the differences are small and usually at or below the level of the statistical errors.

\subsection{Chemical analysis}

\subsubsection{SP_ACE}

For the chemical analysis of our spectra we use the newly developed code SP_ACE (Stellar Parameters and Chemical Abundances Estimator; Boeche \& Grebel 2015), an evolution of the RAVE chemical abundance pipeline (Boeche et al. 2011). In short, the code employs a full-spectrum-fitting technique to derive stellar atmospheric parameters and chemical abundances by means of a $\chi^{2}$-minimization procedure. The reference spectra are generated with a library of general curve-of-growth functions for a given line-list within the desired wavelength range. We point the interested reader to Boeche \& Grebel (2015) for a detailed description of the method, the code itself, and its performance on synthetic and real spectra.

A major advantage of fitting entire wavelength regions is that the amount of information extracted from the data is improved compared to an individual line analysis, specifically because blended absorption features of the same and of different species can be incorporated in the analysis. As a consequence, reliable results can be obtained for many chemical elements, even in spectra of low resolution and/or low signal-to-noise (e.g. Kirby et al. 2008; Caffau et al. 2013; Conroy et al. 2014; Choi et al. 2014; or Hendricks et al. 2014a). Another advantage is that the iterative fitting procedure of the full spectrum within SP_ACE is able to take into account the knowledge about individual absorption features for the continuum placement, which otherwise can have an unwanted biasing effect on the derived continuum level and therefore on the derived chemical abundances (e.g. Kirby et al. 2008). The limited luminosity (and hence distance) range in which classical, high-resolution, and high signal-to-noise chemical abundance analysis can be carried out can thus be expanded to extragalactic targets, such as MW satellites or unresolved systems in the Local Group.

While it seems generally possible to perform a chemical analysis with $\sigma[\mathrm{X} / \mathrm{Fe}] \leq 0.2$ even on low-resolution spectra with $R \sim 3000$ (e.g. Kirby et al. 2008; Conroy et al. 2014), SP_ACE is theoretically capable to determine robust abundances for $R \geq$ 2000 and is specifically tested between $R=2000-20000$. Therefore, we degrade our spectra slightly to a resolution of $R \approx 16000$ to place them on the well-calibrated regime of SP_ACE and also to obtain a higher signal-to-noise per resolution element. For the fainter stars, this improves the proper placement of the continuum and reduces the confusion between noise and weak absorption lines within the fitting procedure. Nonetheless, we test the consistency of the derived chemical abundances to results obtained using the original $R \approx 28000$ resolution, and find low scatter and no systematic changes in the results (see Sect. 3.2.3). 
The most critical problem for an automated, full-spectrum analysis routine is its susceptibility to artifacts related to fitting sky and cosmic ray residual features, which may lead to problems in the continuum placement. Spectra of lower signal-tonoise, as we analyze here, are specifically vulnerable to these points. To address this issue, we visually inspect the model fit to each spectrum and remove individual pixels or wavelength areas from the fit in cases where they can be clearly identified as the origin for a mismatch. This "visual mask" is iteratively refined for each of our targets until we either obtain a satisfactory fit or no obvious cause for a poor fit can be identified. In the latter case, the spectra will have large $\chi^{2}$-values, and we treat their outcome with care. On top of this, we reject absorption features that are problematic to model (in our wavelength range, e.g., $\mathrm{H}_{\alpha}$ or the Telluric feature blueward of $6320 \AA$ ).

In summary, we supply four different pixel masks to SP_ACE which define wavelength regions to ignore in the analysis. While use of the visual mask and the regions containing the Littrow ghost feature are mandatory in order to obtain reliable results, the cosmic-ray and sky masks only have a negligible impact on our results. We only use the cosmic-ray and sky masks later to test the robustness of the chemical analysis against possible inaccuracies in the correction for sky emission and cosmic ray contamination during the process of data reduction.

\subsubsection{Atmospheric parameters}

Generally, SP_ACE is capable to derive stellar atmospheric parameters within the fitting process. However, given the fairly low signal-to-noise of our spectra, a more robust result can be achieved when $T_{\text {eff }}$ and $\log g$ are estimated from multi-band photometry. Specifically, we use $V$ and $I$ magnitudes from our HST photometry and derive temperatures $T_{\text {eff }}$ and bolometric corrections from the empirical equations given in Alonso et al. (1999). For the reddening, we adopt $E(B-V)=0.08$ from Greco et al. (2007) and apply an object-specific transformation described in Hendricks et al. (2012; specifically their Eqs. (3) and (4)) to obtain $E(V-I)=0.11$, suited for a red giant of $[\mathrm{Fe} / \mathrm{H}]=-1.0 \mathrm{dex}, T_{\text {eff }}=4250 \mathrm{~K}$, and $\log g=1.5$. Adopting a standard reddening law $\left(R_{V}=3.1\right)$, we furthermore obtain a $V$-band extinction of $A_{V}=0.248$. We assume no significant differential reddening within our field-of-view.

For surface gravities, $\log g$, we then used the standard relation:

$\log g=\log g_{\odot}+\log \left[\left(\frac{T_{\mathrm{eff}}}{T_{\mathrm{eff}, \odot}}\right)^{4}\left(\frac{M}{M_{\odot}}\right)\left(\frac{L_{\mathrm{bol}}}{L_{\mathrm{bol}, \odot}}\right)^{-1}\right]$.

Here, we apply a distance modulus of $\mu_{0}=20.84$, adopted from Pietrzyński et al. (2009). In order to account for a possibly large age spread among stars in our sample, we used Dartmouth Isochrones (Dotter et al. 2008) and a simple linear age-metallicity relation to assign individual masses to our targets. Specifically, we obtain a variation of stellar mass from $1.27 M_{\odot}$ for stars with $[\mathrm{Fe} / \mathrm{H}]=-1.0$ to $0.79 M_{\odot}$ for a stars with $[\mathrm{Fe} / \mathrm{H}]=-2.0$. Finally, the micro-turbulence is assumed to be a function of $T_{\text {eff }}$ and $\log g$, and is calculated with a third-order polynomial given in Boeche \& Grebel (2015). The atmospheric parameters are iteratively determined with updated stellar metallicities from SP_ACE.

\subsubsection{Consistency tests}

As outlined in the previous sections, several of the assumptions we make during data reduction and analysis may have systematic effects on the derived chemical abundances. Therefore, we perform a series of consistency tests to assess the robustness of our analysis against these factors. Specifically, we test:

a) the results obtained using the full wavelength range (minus the mandatory pixel masks) compared to the outcome using only one of the five individual orders. The motivation of this test is that each order underwent an individual reduction and extraction process, including wavelength calibration, throughput calibration, (tweaked) sky subtraction, and continuum fit, and therefore may display individual systematic biases.

b) the results obtained using the full wavelength range compared to the outcome when additional sky emission regions are flagged out, using the sky-mask. The test is motivated by the necessity for a tweaked sky subtraction to compensate for inaccurate throughput calibration.

c) the results obtained using the full wavelength range compared to the outcome when additional cosmic ray regions are flagged out, using the cosmic-ray mask. The test is motivated by the necessity for a cosmic-ray subtraction prior to spectrum extraction, due to the long individual exposure times.

d) the results obtained using a slightly degraded resolution of $R \approx 16000$, compared to the original $R \approx 28000$.

e) the results obtained using artificially altered atmospheric parameters by $\Delta T_{\text {eff }}=+200 \mathrm{~K}$.

f) the results obtained using artificially altered atmospheric parameters by $\Delta \log g=+0.3$.

Results for $[\mathrm{Fe} / \mathrm{H}]$ are shown in Fig. 5. We did not find any global systematic bias in the derived abundance for any of the tested scenarios (except for the artificial temperature and gravity variations). We also did not find any trends with metallicity, and the scatter for all cases is typically well within \pm 0.2 dex. Importantly, all five individual orders yield consistent results, but with some scatter caused mostly by the limited line information within the smaller wavelength range. A change in $T_{\text {eff }}$ by $+200 \mathrm{~K}$ results in $\Delta[\mathrm{Fe} / \mathrm{H}] \approx-0.2$ dex for stars with low metallicity and decreases to smaller offsets towards more metalrich stars. A change in $\log g$ by +0.3 has practically no effect amongst metal-deficient stars, and yields $\Delta[\mathrm{Fe} / \mathrm{H}] \approx-0.1$ for stars of high metallicity.

\subsubsection{Uncertainty estimates}

Statistical uncertainties are estimated within SP_ACE and expressed as a separate upper and lower limit of the derived $[\mathrm{X} / \mathrm{H}]$ elemental abundances $\left([\mathrm{X} / \mathrm{H}]_{+} \text {and }[\mathrm{X} / \mathrm{H}]_{-}\right)^{3}$. Here, the upper and lower limit express the $68 \%$ probability with no guarantee that the semi-error expresses the $34 \%$ limit (see Boeche \& Grebel 2015 for more details). For the errors in relative abundances $([\mathrm{X} / \mathrm{Fe}])$, we simply calculate the semi-errors as quadratic sum of the upper and lower semi-errors (e.g., $\sigma[\mathrm{X} / \mathrm{Fe}]_{+}{ }^{2}=\sigma[\mathrm{X} / \mathrm{H}]_{+}{ }^{2}+\sigma[\mathrm{Fe} / \mathrm{H}]_{+}{ }^{2}$ ) and therefore overestimate the error slightly by ignoring the covariance terms between $[\mathrm{Fe} / \mathrm{H}]$ and $[\mathrm{X} / \mathrm{H}]$ in a full-spectrum-fitting approach. Uncertainties for $[\mathrm{Fe} / \mathrm{H}]$ range from $\leq 0.1$ dex to $\sim 0.3 \mathrm{dex}$, depending on the brightness and the metallicity of the star. For

3 When the upper or lower abundance limit falls beyond the internal SP_ACE abundance grid, a null-value is reported. 

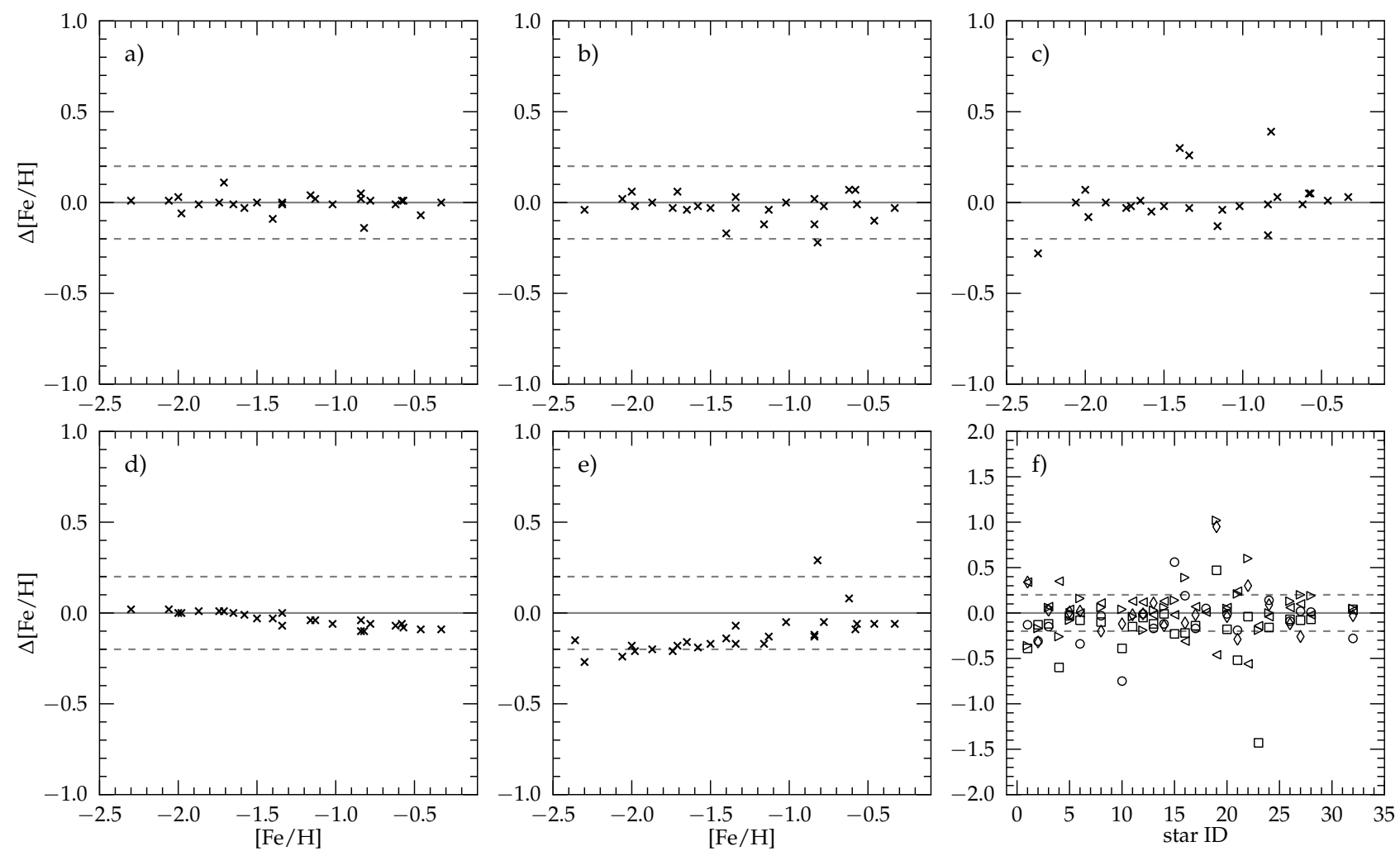

Fig. 5. Consistency tests for chemical abundances derived with SP_ACE. All plots show the difference between the derived [Fe/H] and various changes to the spectrum as described in the text: a) with cosmic ray regions flagged out; b) with sky emission regions flagged out; c) adopting the initial resolution of $R=28000 ; \mathbf{d}) \Delta \log g=0.3 ; \mathbf{e}) \Delta T_{\text {eff }}=200 K$; f) all five orders analyzed separately (orders 52, 53, 54, 55, 56: circle, diamond, square, left-handed triangle, right-handed triangle). Given the limited wavelength range and the limited chemical information, not every individual order in each spectrum provides a result. Additionally, some orders in some stars are more affected by the different pixel masks and hence might show larger offsets.

$[\alpha / \mathrm{Fe}]$ (where $\alpha=\mathrm{Ca}, \mathrm{Si}$, or Ti) we obtain similar but somewhat larger errors. Iron-peak element $(\mathrm{V}, \mathrm{Cr}, \mathrm{Co}, \mathrm{Ni})$ ratios typically show uncertainties as small as $[\mathrm{Fe} / \mathrm{H}]$. Finally, the statistical uncertainties are included with some systematic error as discussed in the previous section, which limits the final accuracy of our results to $\delta[\mathrm{X} / \mathrm{H}] \sim 0.1 \mathrm{dex}$, even for the brightest targets.

Astrometric and photometric information, as well as the derived chemical abundances with their uncertainties for all our targets are given in the Appendix (Tables A.1-A.3, respectively).

\section{Results}

\section{1. $[\mathrm{Fe} / \mathrm{H}]$, radial velocities and membership likelihood}

Due to its position close to the center of Fornax, stars within the tidal radius of $\mathrm{H} 4$ are severely contaminated by field stars of the galaxy. A membership likelihood determination and a clear assignment of our program stars to either the cluster or the field is crucial because the chemical properties of $\mathrm{H} 4$ compared to the field is a major goal of this study.

Here, we use three observed properties to determine the membership likelihood of a target star to the cluster H4: The star's proximity to the cluster center $\left(r_{\mathrm{GC}}\right)$, its radial velocity $v$, and its iron abundance $[\mathrm{Fe} / \mathrm{H}]$ (here $m$ ). Generally, the probability that a given star with properties $\{P\}$ is a member of the cluster $\mathrm{H} 4$ is given by

$P_{\mathrm{H} 4}(\{P\})=\frac{N_{*}(\{P\} \mid \mathrm{H} 4)}{N_{*}(\{P\} \mid \mathrm{H} 4)+N_{*}(\{P\} \mid \text { field })}$, where $N_{*}(\{P\} \mid \mathrm{H} 4)$ is the number of $\mathrm{H} 4$ members with properties $\{P\}=p_{1}, p_{2}, \ldots p_{i}$ and similarly $N_{*}(\{P\} \mid$ field $)$ denotes the number of field stars which share the same parameter space.

Using our HST photometric catalog, we first obtain an initial membership probability from the stellar density profile of the cluster, which we fit with a King profile $\left(K\left(r_{\mathrm{GC}}\right)\right.$; King 1966), and extrapolate the function inwards to the unresolved center for radii $\leq 12^{\prime \prime}$ (see Fig. 6). Notably, the contamination rate is high both at radii beyond half the tidal radius $(\geq 50 \%)$ and near the cluster center $(\approx 20 \%)$.

The fraction of stars above the uniform background compared to the background level $\left(\rho_{\mathrm{b}}\right)$ itself yields the membership probability $P_{\mathrm{H} 4}\left(r_{\mathrm{GC}}\right)$ for a given star with distance $r_{\mathrm{GC}}$ to the cluster center:

$$
P_{\mathrm{H} 4}\left(r_{\mathrm{GC}}\right)=\frac{N_{*}\left(r_{\mathrm{GC}} \mid \mathrm{H} 4\right)}{N_{*}\left(r_{\mathrm{GC}} \mid \mathrm{H} 4\right)+N_{*}\left(r_{\mathrm{GC}} \mid \text { field }\right)}=\frac{K\left(r_{\mathrm{GC}}\right)}{K\left(r_{\mathrm{GC}}\right)+\rho_{\mathrm{b}}} .
$$

The unfortunate circumstance of heavy contamination is somewhat compensated by the fact that $\mathrm{H} 4$ members show a distinctly different radial velocity and $[\mathrm{Fe} / \mathrm{H}]$ compared to the field stars. The radial velocity of the cluster has been measured in integrated-light studies by Larsen et al. (2012) and Dubath et al. (1992), who consistently report values of 46.2 and $47.2 \mathrm{~km} \mathrm{~s}^{-1}$, respectively. Therefore $\mathrm{H} 4$ 's radial velocity is lower by $\sim 9 \mathrm{~km} \mathrm{~s}^{-1}$ compared to the mean galactic motion of Fornax, which approximately corresponds to the velocity dispersion of the galaxy at this metallicity (see Hendricks et al. 2014b). Additionally, H4's metallicity is measured around $[\mathrm{Fe} / \mathrm{H}]=$ -1.4 (Larsen et al. 2012; Strader et al. 2003), and is therefore 


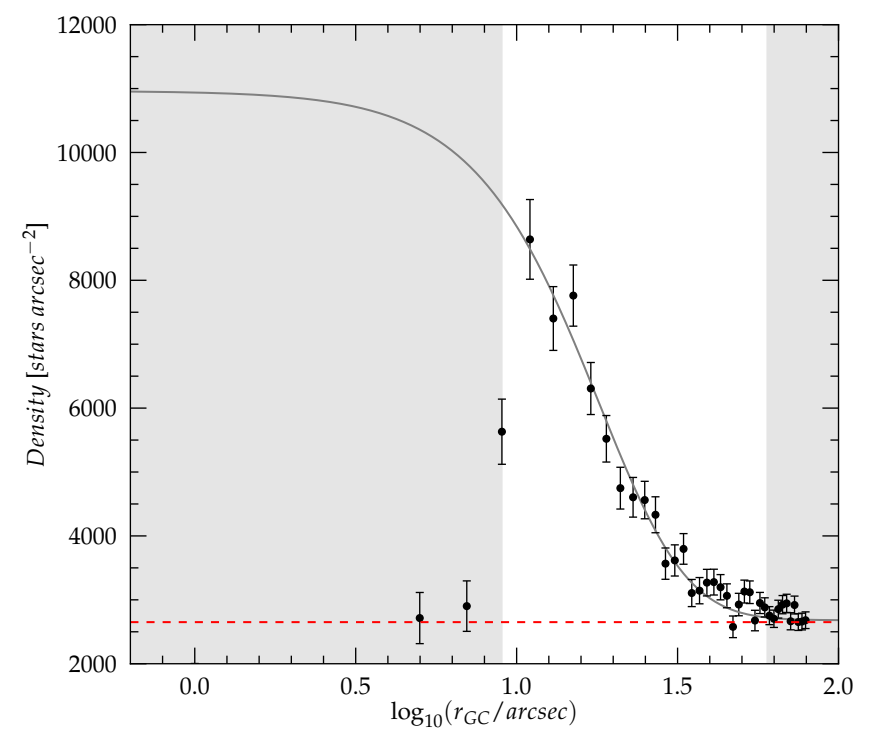

Fig. 6. Stellar density profile of the GC H4 from HST photometry. The gray line is a King-profile fit to the data at radii beyond $r_{\mathrm{GC}}=12^{\prime \prime}$. The red line indicates the background level that we determine from the stellar density outside the tidal radius of the cluster. The white area in the figure shows the radial zone from which we pick our targets. At smaller radii, the cluster is not resolved with ground-based telescopes, and the probability that a star at larger radii is a member of the system is very low.

significantly lower than the galaxy average of $\sim-0.9$ dex in the central part of Fornax.

When we combine the metallicity $m$, the velocity $v$, and the proximity to the cluster center $r_{\mathrm{GC}}$, we can put a tight membership probability for our stars:

$$
\begin{aligned}
P_{\mathrm{H} 4}\left(r_{\mathrm{GC}}, v, m\right) & =\frac{N_{*}\left(r_{\mathrm{GC}}, v, m \mid \mathrm{H} 4\right)}{N_{*}\left(r_{\mathrm{GC}}, v, m \mid \mathrm{H} 4\right)+N_{*}\left(r_{\mathrm{GC}}, v, m \mid \text { field }\right)} \\
& =\frac{K\left(r_{\mathrm{GC}}\right) \times p(v, m \mid \mathrm{H} 4)}{K\left(r_{\mathrm{GC}}\right) \times p(v, m \mid \mathrm{H} 4)+\rho_{\mathrm{b}} \times p(v, m \mid \text { field })} .
\end{aligned}
$$

Here, $p(v, m \mid \mathrm{H} 4)$ denotes the probability for an $\mathrm{H} 4$ member star to display the properties $v$ and $m$. Similarly, and $p(v, m \mid$ field $)$ is the equivalent expression for field stars.

We extract the chemical and dynamical properties for the contaminating field stars from the catalog provided in Battaglia et al. (2008), which provides calcium-triplet metallicities and radial velocities for nearly 1000 stars. To take into account radial variations of these properties within Fornax, we only consider 406 objects which are located at similar radii to the cluster, and specifically stars with $r \leq 0.3^{\circ}$.

We do not know the exact properties of $\mathrm{H} 4$ given that all information is derived from integrated-light analysis. With the risk of systematic misplacement of its actual properties, we assume that all $\mathrm{H} 4$ members fall within certain limits of $[\mathrm{Fe} / \mathrm{H}]$ and $v$ where $p(v, m \mid \mathrm{H} 4)=1$, but do not have a preferred position within these intervals. Consequently, there is a zero likelihood to find a member outside these limits. We set the allowed parameter space for $\mathrm{H} 4$ members between $-1.65 \leq[\mathrm{Fe} / \mathrm{H}] \leq-1.3$ and $42.5 \leq v \leq 52.5 \mathrm{~km} \mathrm{~s}^{-1}$, constrained by previous integratedlight measurements that take into account their measurement error and a possible systematic bias in the methodology. For the velocities, we additionally take into account $\mathrm{H} 4$ 's intrinsic velocity dispersion of $\sim 4.5 \mathrm{~km} \mathrm{~s}^{-1}$ (Larsen et al. 2012; Dubath et al. 1992).

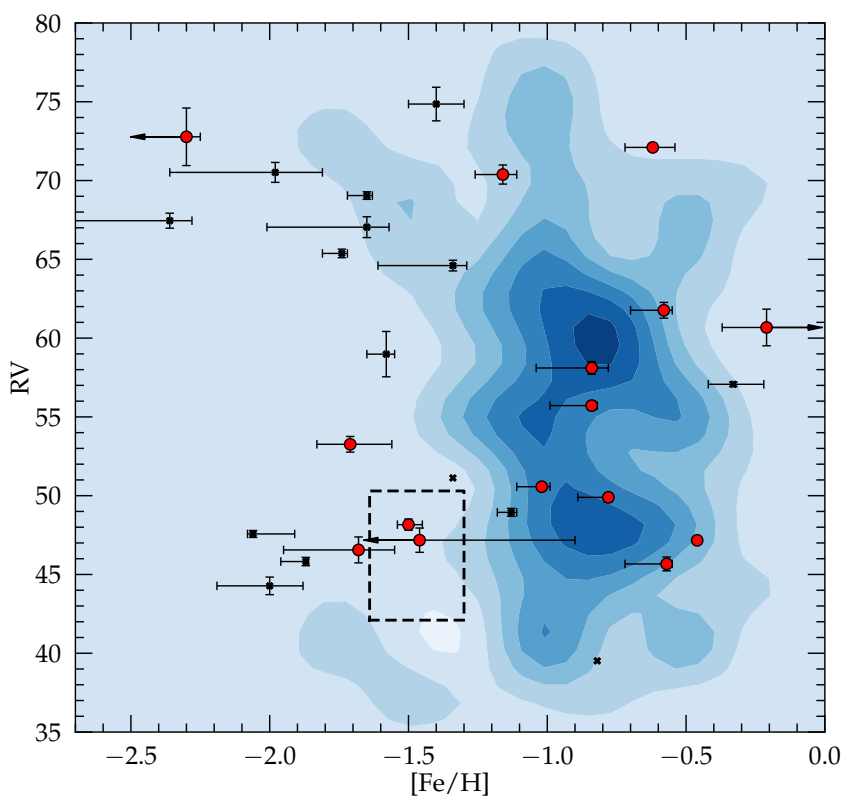

Fig. 7. Radial velocity and $[\mathrm{Fe} / \mathrm{H}]$ of our targets stars overplotted to the field star distribution, visualized as a linearly-scaled density distribution of arbitrary units (blue contours). Stars within the tidal radius of $\mathrm{H} 4$ are shown in red. Importantly, three of them group within the parameter space where we expect cluster members (black box). Black symbols are targets outside the tidal radius, which are purposefully selected to be metal-poor and therefore are not representative of the actual field star distribution.

For the field stars, the distributions of $[\mathrm{Fe} / \mathrm{H}]$ and the radial velocities are not Gaussian functions nor are they any other evident analytical shape. We therefore abandon the attempt to model the complex distribution in a combined parameter space. Instead, we assess the contamination fraction $p(v, m \mid$ field $)$ empirically from the fraction of field stars that fall within the allowed parameter box of $\mathrm{H} 4$ and find $p(v, m \mid$ field $)=0.020$. Under these assumptions - and if its Fe and $v$ identifies a star as a potential cluster candidate - the probability for each of our targets to be an actual member of the cluster, becomes

$P_{\mathrm{H} 4}\left(r_{\mathrm{GC}}, v, m\right)=\frac{K\left(r_{\mathrm{GC}}\right)}{\rho_{\mathrm{b}} \times p(v, m \mid \text { field })+K\left(r_{\mathrm{GC}}\right)}$.

Finally, we use a bootstrapping technique to estimate the uncertainty for $P_{\mathrm{H} 4}\left(r_{\mathrm{GC}}, v, m\right)$ by constructing a number of equally sized samples where each individual entry is altered randomly within its uncertainties in $v$ and $m$.

In Fig. 7, we show the derived $[\mathrm{Fe} / \mathrm{H}]$ abundances and velocities on top of the underlying field star distribution. Most of the stars we selected from within the tidal radius of $\mathrm{H} 4$ resemble typical properties of field stars in Fornax, with high metallicities $([\mathrm{Fe} / \mathrm{H}] \geq-1.2)$ and velocities of $\sim 45-70 \mathrm{~km} \mathrm{~s}^{-1}$. From the few outliers, one is very metal-poor $([\mathrm{Fe} / \mathrm{H}] \leq-2.0 \mathrm{dex})$ and with high radial velocity. This combination may be typical given that previous studies already detected a trend for more extreme velocities amongst metal-deficient stars in the galaxy (Battaglia et al. 2006; Hendricks et al. 2014b). The remaining four all have $[\mathrm{Fe} / \mathrm{H}] \approx-1.5$, and three of them show almost identical radial velocities of $47.2,48.2$, and $46.6 \mathrm{~km} \mathrm{~s}^{-1}$. For the last candidate we measure a similar metallicity but a radial velocity about two times larger than the intrinsic dispersion within H4. Strikingly, two of the stars that fall within the acceptable parameter space are also located closest to the center of $\mathrm{H} 4$, which strengthens the assumption that they are members of the cluster. For them, we 
obtain a membership likelihood of $99.2 \pm 0.4 \%$ (target r_0010) and $97.7 \pm 1.2 \%$ (target $r \_0016$ ), respectively, but the third star (target b_0018) with larger $r_{\mathrm{GC}}$ is still a $71.0 \pm 11.0 \%$ member of H4. Given a membership likelihood of at least 2-3 sigma for our best candidates, we will assume that these stars are true cluster members in the following chemical analysis and discussion.

The remaining targets outside the tidal radius of $\mathrm{H} 4$ have been picked deliberately from the sparse metal-poor tail of the galaxy, and therefore their $[\mathrm{Fe} / \mathrm{H}]-v$ distribution does not reflect the dominant distribution of the field star population.

Our chemical analysis yields iron abundances for 30 targets, 15 of which are located within the tidal radius of $\mathrm{H} 4$. Of these stars, three "clump" around the fiducial cluster properties. Given this small number, our sample does not allow for an individual estimate of the cluster's chemical or dynamical properties, and in contrast - we adopted these mean cluster parameters from the literature to identify member stars. From our three likely members, we find a weighted (by error and membership likelihood) mean metallically for our member stars of $[\mathrm{Fe} / \mathrm{H}]=-1.56 \mathrm{dex}$, slightly lower than the values obtained from integrated-light studies. This may not be surprising when we consider that most of the contaminants in integrated-light spectra are undoubtedly more metal-rich than the cluster itself. With a similar explanation, our bona fide members have weighted mean radial velocity of $47.6 \mathrm{~km} \mathrm{~s}^{-1}$, marginally higher than the integrated-light estimates.

\subsection{Alpha-elements}

The alpha-elements in stellar atmospheres analyzed with respect to iron reveal the fraction of SN Ia contributions to the star forming material and hence are an indicator for the enrichment efficiency of the galactic environment. The $[\alpha / \mathrm{Fe}]$ abundance ratio is known to be distinctively different in the field star population of dwarf galaxies compared to the MW.

In Fig. 8 we show the results for the alpha-elements $\mathrm{Ca}$ and Ti as a function of the stars' iron abundances. For both elements, we find a clear sequence with metallicity. While field stars with $[\mathrm{Fe} / \mathrm{H}] \leq-1.8$ tend to have $[\alpha / \mathrm{Fe}]$ above solar level, this ratio drops continuously to clearly sub-solar values for $[\mathrm{Fe} / \mathrm{H}] \approx-1.3$ and higher. Clearly, our stars show an early depletion in both elements compared to the MW halo. This trend does not allow for a knee in the alpha-iron evolution at $[\mathrm{Fe} / \mathrm{H}]$ significantly higher than $-2.0 \mathrm{dex}$, which confirms previous findings by Hendricks et al. (2014a) and later Lemasle et al. (2014), and indicates a low chemical enrichment efficiency within the Fornax dSph galaxy. One star falls out of this general scheme by showing high $[\alpha / \mathrm{Fe}]$ for $\mathrm{Ca}$ and $\mathrm{Ti}$, although it is the star with the highest $[\mathrm{Fe} / \mathrm{H}]$ in our sample. By that, it better resembles the characteristics of MW disc stars. Therefore, it is possible that this star is a Galactic interloper that does not belong to the Fornax dwarf spheroidal.

While two of the $\mathrm{H} 4$ members in our sample are too faint to determine reliable alpha-abundances, one star (r_0010 with $99.2 \%$ membership likelihood) is one of our brightest targets and we can derive precise abundances for $\mathrm{Ca}$, Ti, and, with larger uncertainties, Si. For this star, we measure low $[\alpha / \mathrm{Fe}]$ ratios for all three elements. In detail, we find $[\mathrm{Ca} / \mathrm{Fe}]=+0.05_{-0.07}^{+0.09},[\mathrm{Ti} / \mathrm{Fe}]=$ $-0.27 \pm 0.23$, and $[\mathrm{Si} / \mathrm{Fe}]=-0.35 \pm 0.34$, resulting in an average $[\alpha / \mathrm{Fe}]=-0.19 \pm 0.14$. Given the high membership likelihood to $\mathrm{H} 4$ and the small uncertainties on our abundances, this is a strong indication that $\mathrm{H} 4$ is an alpha-depleted GC with a combined $[\alpha / \mathrm{Fe}]$ possibly at sub-solar level.

To this point, the only existing measurement of alphaelements in H4 comes from Larsen et al. (2012) and is based on
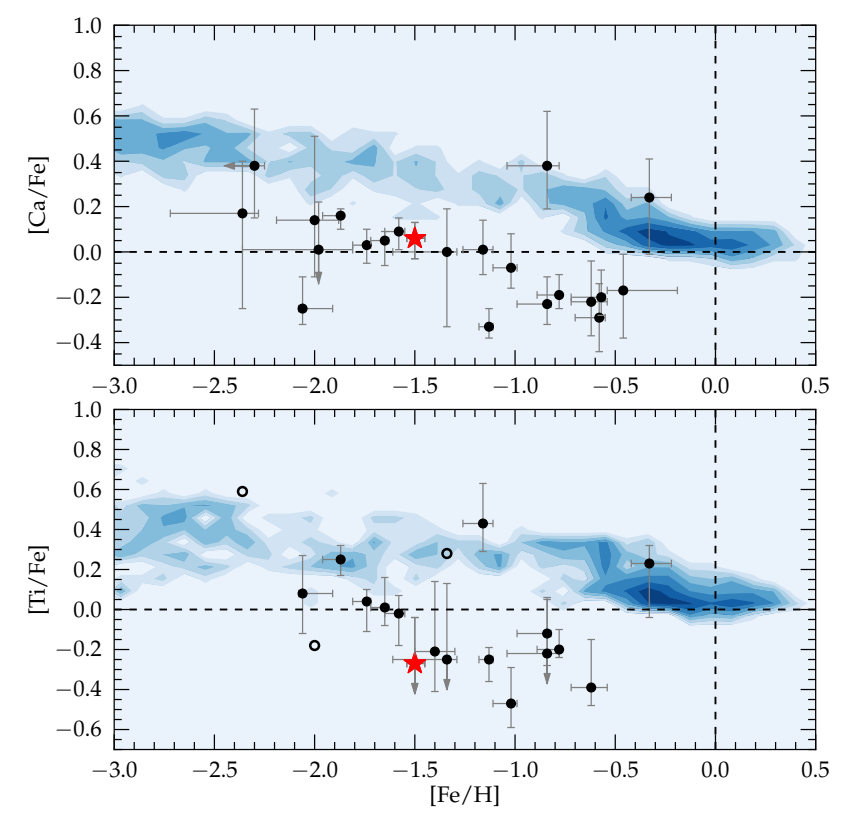

Fig. 8. Chemical evolution of the alpha-elements $\mathrm{Ca}$ (upper panel) and Ti (lower panel) as a function of $[\mathrm{Fe} / \mathrm{H}]$. Black dots show the field stars in our sample and the red star highlights the GC H4 star. For visual comparison, the pattern of MW disc and halo stars are shown as a logarithmically-scaled number density distribution of arbitrary units (data from Venn et al. 2004 and Roederer et al. 2014). For both elements, we observe a clear sequence with $[\mathrm{Fe} / \mathrm{H}]$, and in both cases the $\mathrm{H} 4$ member falls on top of this sequence and does not agree with the chemical abundance pattern seen in the MW. Open circles indicate objects for which SP_ACE could not find upper and lower abundance limits, either due to the low quality of the spectrum or because its chemical parameters fall close to the boundary of the allowed abundance grid.

integrated-light spectroscopy of the cluster. These authors find $[\mathrm{Ca} / \mathrm{Fe}]=+0.13 \pm 0.07$ and $[\mathrm{Ti} / \mathrm{Fe}]=+0.12 \pm 0.05$, which is somewhat larger than our results from an individual member star. The discrepancy might be partially explained with the measurement errors of the respective analyses. Furthermore, it is important to recall the high contamination of more than $20 \%$ within the $5^{\prime \prime}$ slit, which has been used to obtain the integrated-light spectrum. These authors carefully try to minimize the impact of this contamination by evaluating the spectrum at different positions, and therefore at different cluster radii. Unfortunately, the options to detect and correct for contamination effects are limited, because the contamination fraction does not change by more than $10 \%$ within the central $5^{\prime \prime}$ of the cluster. As a consequence, it is difficult to reconstruct the actual underlying population mix because the contaminating field stars show a broad range of ages and metallicities. Therefore, even with a careful approach, a small bias between results obtained from integratedlight and individual stars can be expected. Overall, however, both studies agree that $\mathrm{H} 4$ has lower $[\alpha / \mathrm{Fe}]$ than similar metallicity Milky Way GC and field stars.

\subsubsection{The full picture: Co-evolution of field stars and GCs in Fornax}

Important insights can be obtained when the alpha-abundances of all GCs in Fornax are viewed in the context of the field star enrichment in the galaxy. If we combine literature samples of $[\mathrm{Ca} / \mathrm{Fe}]$ measurements for field stars (43 stars from Lemasle et al. 2014, 85 stars from Letarte et al. 2010) with our own sample from this work (21 stars), we obtain a well defined and coherent alpha-element evolution sequence (see Fig. 9). The data 


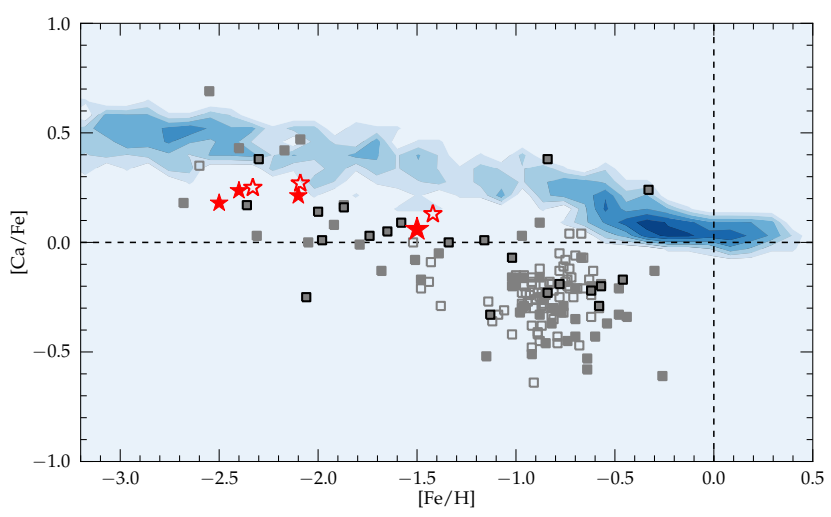

Fig. 9. Fornax displays a different chemical enrichment pattern from the MW. This is seen in the alpha-element evolution of the field stars and the GCs, which show a coupled chemical enrichment. Blue contours show the pattern of MW field stars from Venn et al. (2004) and Roederer et al. (2014). Fornax field stars come from Letarte et al. (2010; open gray), Lemasle et al. (2014; filled gray), and this work (filled gray with black edge). Star symbols show the location of the metal-deficient GCs H1, H2, H3 (filled, small red stars; Letarte et al. 2006), H3, H4, H5 (open red stars; Larsen et al. 2012) and the measurement for $\mathrm{H} 4$ presented in this work (filled large red star).

clearly show that for $[\mathrm{Fe} / \mathrm{H}]$ below approximately $-2.0 \mathrm{dex}$, field stars are alpha-enhanced and share the typical properties of MW halo stars (see also Tafelmeyer et al. 2010 for one star at $[\mathrm{Fe} / \mathrm{H}]=-3.66$ and $[\mathrm{Ca} / \mathrm{Fe}]=+0.48)$. Towards higher metallicities, the $[\alpha / \mathrm{Fe}]$ ratio drops and evolves from super-solar to sub-solar values around $[\mathrm{Fe} / \mathrm{H}] \approx-1.5$. Although there are no stars in common between the individual field star samples, there is an excellent agreement between the observed properties for all respective metallicities.

The four metal-poor GCs in Fornax are all moderately enhanced in $[\alpha / \mathrm{Fe}]$, and have values comparable to clusters found in the halo of the MW. However, the metal-poor Fornax GCs may lie somewhat below the average plateau found in the MW. In detail, for three of the metal-deficient clusters in Fornax, $[\mathrm{Ca} / \mathrm{Fe}]$ has been measured from three individual stars in each cluster by Letarte et al. (2006). These authors find alphaenhanced values for all three systems with average values of $+0.18 \pm 0.09,+0.22 \pm 0.06,+0.24 \pm 0.03$ for $\mathrm{H} 1, \mathrm{H} 2$, and $\mathrm{H} 3$, respectively. Additionally, Larsen et al. (2012) also derive $[\mathrm{Ca} / \mathrm{Fe}]$ from integrated-light analysis for the metal-poor GCs H3 and H5 and find $+0.25 \pm 0.07$ and $+0.27 \pm 0.05$.

Taking the information from field stars and GCs in Fornax together, two important consequences arise. First, it is very likely that the cluster $\mathrm{H} 4$ does have a significantly lower $[\alpha / \mathrm{Fe}]$ abundance ratio than the rest of the GC population in Fornax. Second, the clusters in Fornax follow the $[\alpha / \mathrm{Fe}]$ sequence of the field stars and clearly disagree with with the enrichment pattern of the MW halo. Therefore, there is strong evidence that the chemical enrichment of GCs and field stars in the Fornax dSph is coupled, and that the clusters trace the chemical signatures of the field star population in their host. We will discuss the consequences of such a scenario for other alpha-depleted GCs in Sect. 5.1.

The evident chemical enrichment pattern also highlights the star formation differences between Fornax and the MW. In the MW, $[\alpha / \mathrm{Fe}]$ declines for the field stars but not the GCs, which means that the GCs with, e.g., $[\mathrm{Fe} / \mathrm{H}] \approx-0.3$ possibly formed before the field stars of the same metallicity. Such a scenario does not appear to be the case with Fornax where the field stars and GCs follow each at metallicities exceeding the location of the $[\alpha / \mathrm{Fe}]$ knee.
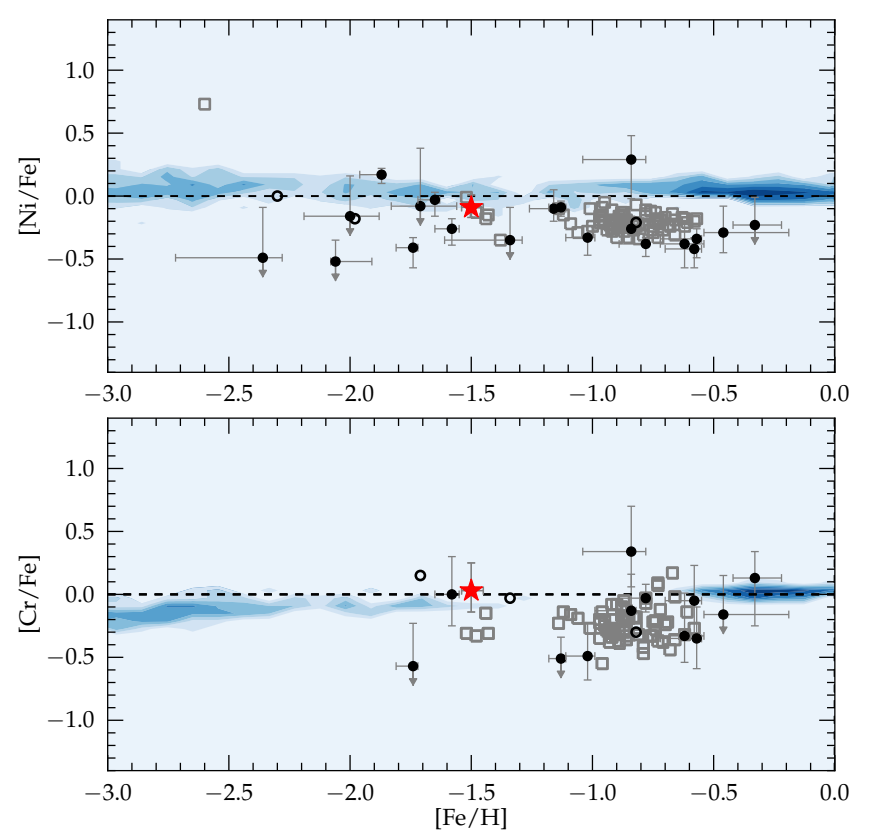

Fig. 10. $[\mathrm{Ni} / \mathrm{Fe}]$ and $[\mathrm{Cr} / \mathrm{Fe}]$ as a function of $[\mathrm{Fe} / \mathrm{H}]$. As in Fig. 8, black dots show the field stars in our sample and the red star highlights the GC H4 star. Contours show the pattern of MW disc and halo stars (data from Venn et al. 2004; Roederer et al. 2014; Reddy et al. 2003, and Bensby et al. 2003). Open circles indicate objects for which SP_ACE could not find upper and lower abundance limits. Gray open squares are Fornax field star measurements from Letarte et al. (2010).

\subsection{Iron-peak elements}

It is thought that most iron-peak elements (Sc to $\mathrm{Zn}: 21 \leq Z \leq$ 30, excluding Ti which behaves like an alpha-element) descend from similar nucleosynthetic pathways. While at early times, massive stars and their subsequent $\mathrm{SNe}$ II explosions are the only production resource, $\mathrm{SNe}$ Ia become the dominant contributor later on, when low-mass stars had time to sufficiently evolve. Although they form a common family of elements, the exact formation channels for individual species are not clear, and in most cases there is no simple scaling with the Fe abundance. For a large fraction of stars in our sample, we obtain abundances for several iron-peak elements to compare with the Galactic trends. The results are shown in Fig. 10.

Nickel. The $[\mathrm{Ni} / \mathrm{Fe}]$ ratio is generally underabundant by $\sim 0.2$ dex compared to solar and MW abundance ratios. Such low abundances have been previously observed in Fornax (Letarte et al. 2010; Lemasle et al. 2014) and also in Sagittarius (e.g., Carretta et al. 2010a; Sbordone et al. 2007). The LMC displays a larger scatter in Ni above and below solar (Pompéia et al. 2008). Our results seem to indicate that the sub-solar $[\mathrm{Ni} / \mathrm{Fe}]$ abundance ratio, which has been perviously found for stars in satellite systems between $[\mathrm{Fe} / \mathrm{H}] \approx-0.5 \mathrm{dex}$ and $[\mathrm{Fe} / \mathrm{H}] \approx-1.5 \mathrm{dex}$, continues to even lower metallicities.

Chromium. Our $\mathrm{Cr}$ abundances lie slightly below the solar abundance ratio and the Galactic trend, although few reliable comparison data are available for $[\mathrm{Fe} / \mathrm{H}] \geq-1.5 \mathrm{dex}$. They agree well, however, with previous measurements of Fornax field stars from Letarte et al. (2010) and Lemasle et al. (2014), although only a few lines could be measured and the abundances are statistically not very well constrained.

We additionally measure abundances for the iron-peak elements Sc, V, and Co, for which we generally find slightly subsolar abundance ratios. Unfortunately, there is only very few 
comparison data available for MW stars, and no previous measurements exist for Fornax. These abundances are therefore not shown in Fig. 10, and they are only listed in Table A.3 in the Appendix.

\subsection{The age of $\mathrm{H} 4$}

The relative age of $\mathrm{H} 4$ has been a subject of controversy during the last decades, not least because its detailed chemical composition - and specifically the $[\alpha / \mathrm{Fe}]$ abundance of its stars - has not been known. Using a relatively clean sample of HST photometry, Buonanno et al. (1999) estimated the relative age of $\mathrm{H} 4$ from its photometric offset at different regions in the CMD compared to other clusters in Fornax. From this, the authors found $\mathrm{H} 4$ to be $\sim 3$ Gyr younger than the other four GCs, which are all coeval and resemble typical old ( $\sim 12 \mathrm{Gyr})$, metal-poor MW halo GCs like M92 (Buonanno et al. 1998). However, these results were based on the assumption that all Fornax clusters display a similar chemical mixture, in disagreement with our findings. Later, Strader et al. (2003) used age sensitive - but alpha-insensitive - spectroscopic indices like $\mathrm{H}_{\beta}$ and $\mathrm{H}_{\gamma}$ to constrain relative ages amongst Fornax clusters and found a similar age for $\mathrm{H} 4$ compared to the old systems H2 and H3 (they found, however, indications that $\mathrm{H} 5$ is slightly younger). If $\mathrm{H} 4$ is indeed younger compared to the rest of the GC population, it is not clear how Fornax was able to form this cluster several Gyr later than all of the other more metal-poor clusters in the galaxy. This is specifically intriguing when viewed in the context that similarly young Galactic GCs, such as Ruprecht 106 and Palomar 12, are thought to be accreted from satellite dwarf galaxies (Brown et al. 1997).

Precise relative age estimates of GCs can be obtained when isochrones are fitted to to the resolved main-sequence turn-off (MSTO) region of the cluster. If the chemical composition and the line-of-sight reddening to the cluster are known, the addition of zero-age horizontal branch (ZAHB) model fitting can enable one to achieve a precision well below 1 Gyr (see VandenBerg et al. 2013). However, both the position and shape of the MSTO and the HB luminosity are sensitive to the underlying $[\alpha / \mathrm{Fe}]$ ratio. This degeneracy causes a systematic bias in the derived ages of several Gyr for cases where the detailed composition of the cluster is not known.

Provided with a tight constraint on the $[\alpha / \mathrm{Fe}]$ abundance in $\mathrm{H} 4$, we can now make a new approach to constrain the age of the system and specifically aim to answer the question whether $\mathrm{H} 4$ is younger than the remainder of the population in Fornax. To do so, we follow the general procedure described in VandenBerg et al. (2013). Specifically, we derive synthetic ZAHB loci from the lower envelope of synthetic HB models from Dotter et al. (2007) provided on the Dartmouth-Isochrone database ${ }^{4}$ and fit them to the observed red horizontal branch of the cluster to set the absolute magnitude scale. Then, the age of the cluster is determined by the best fitting Dartmouth isochrones (Dotter et al. 2008) to the turn-off and subsequent subgiant branch region, after a reddening correction has been applied. To minimize the number of field stars in the CMD, we consider only an area within $r_{\mathrm{GC}} \leq 15^{\prime \prime}$ around the cluster center. For all models, we adopt $[\mathrm{Fe} / \mathrm{H}]=-1.5$ and $[\alpha / \mathrm{Fe}]=0.0$, and a correction for reddening and extinction of $E(V-I)=0.11$ and $A_{V}=0.248$ (see Sect. 3.2 for details about the reddening).

In Fig. 11 we show the result. From the position of the ZAHB, we find a distance modulus of $\mu_{0}=20.74 \pm 0.4$, in agreement with previous estimates for the distance to the galaxy itself

\footnotetext{
4 http://stellar.dartmouth.edu/ models/index.html
}

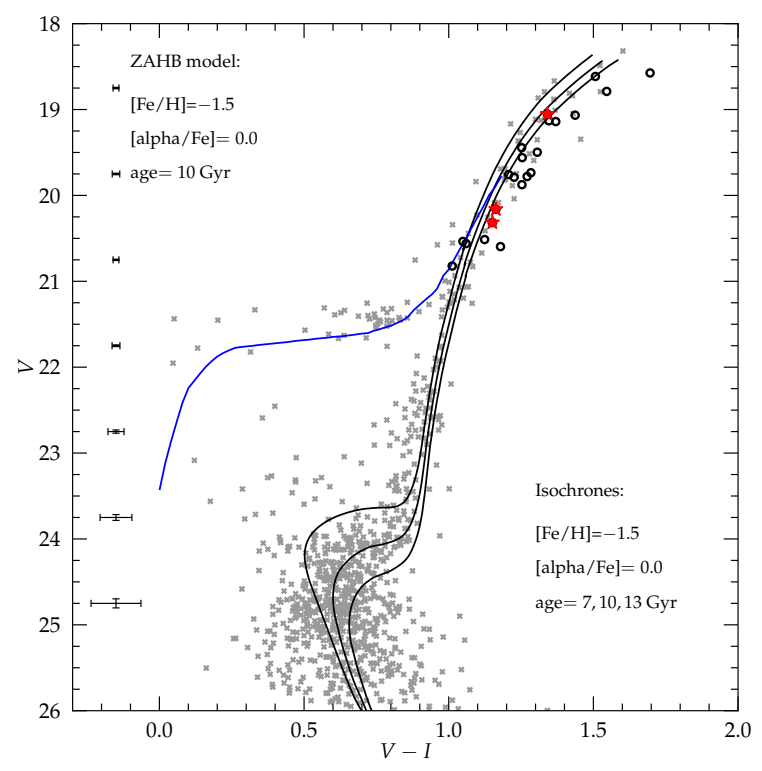

Fig. 11. Color-magnitude diagram of $\mathrm{H} 4$ from our HST photometry. The HST system bands ( $F 555 W$ and $F 814 W$ ) have been transformed to Johnson- Cousins $V$ and $I$ magnitudes (see Sect. 2.2.1 for details). Only stars from the innermost $15^{\prime \prime}$ around the cluster center are shown to minimize the impact of contaminating field stars. Dartmouth Isochrones (Dotter et al. 2008) of 7, 10, and 13 Gyr of age and a 10 Gyr ZAHB model are overplotted to the data. The ZAHB luminosity, however, is not sensitive to age in this regime and therefore the model is representative for all three ages compared here. Red stars indicate the position of the three likely $\mathrm{H} 4$ members in our sample and black circles are other targets with H4's tidal radius. The isochrones give a best fit for ages around $\sim 10 \mathrm{Gyr}$, but uncertainties in reddening and distance to the cluster do not allow a tight age constraint, although the chemical parameters of the cluster are now known.

(e.g., Bersier 2000; Rizzi et al. 2007; Pietrzyński et al. 2009). However, this number is slightly higher than the results of Greco et al. (2007) who find $\mu_{0}=20.53 \pm 0.09$ from RR Lyrae stars in $\mathrm{H} 4$, if $[\mathrm{Fe} / \mathrm{H}]=-1.5$ is adopted.

We obtain the best isochrone fit using an age of $10 \mathrm{Gyr}$, which places $\mathrm{H} 4$ at a younger age compared to the rest of the population. However, this result should be interpreted with caution since the uncertainty is at least $\pm 1 \mathrm{Gyr}$, given the poorly defined MSTO at $V$-band magnitudes around 24.5 and when we consider the still significant fraction of field stars in the CMD with different chemical signatures and ages than the cluster itself. If we further consider inaccuracies in the reddening 5 , an age as old as 12 Gyr, comparable to the other Fornax clusters, seems to be still a conceivable possibility.

\section{Discussion}

\subsection{Origin of alpha-depleted GCs in the halos of larger galaxies}

The evidence that $\mathrm{H} 4$ is an alpha-depleted GC makes it one out of only three known clusters to be depleted at this low metallicity. The other known alpha-depleted clusters are Ruprecht 106 in the MW (Brown et al. 1997; Villanova et al. 2013) and G002 in M31 (Colucci et al. 2014). Importantly, H4 is the only of these clusters which can be clearly associated with a dwarf galaxy, and we may observe in Fornax the first birthplace of a metal-deficient, alpha-depleted GC. The case of Fornax

5 For $\mathrm{H} 4$, the estimates range between $E(B-V)=0.15$ and 0.08 , while Fornax itself has a reddening not higher than $E(B-V)=0.04$ from Schlegel et al. (1998) reddening maps. 
therefore supports speculations which predict similarly depleted clusters in the halo of larger galaxies to originate from dwarf galaxies with low chemical enrichment efficiency (e.g., Colucci et al. 2014).

Following this concept, we can use the properties of accreted GCs to learn about their former host systems. On the one hand, alpha-depleted GCs like Ruprecht 106 and G002 require a host galaxy with a sufficiently low chemical enrichment efficiency to enable significant SNe Ia contribution for stars already at such low metallicities. Given that a galaxy's mass is likely the main driver of its enrichment efficiency (e.g., Matteucci \& Brocato 1990; Tolstoy et al. 2009), the host needs to have stellar masses as low as Fornax or the Sculptor dSph (i.e., a few $10^{7} M_{\odot}$ ), while a system as massive as the Magellanic Clouds can be ruled out. On the other hand, recent studies argue that a large fraction of GCs in the halos of the MW and M31 have not formed in-situ, but instead have been accreted from infalling satellite systems (e.g., Mackey \& Gilmore 2004; Leaman et al. 2013; Mackey et al. 2010; Huxor et al. 2011; Elmegreen et al. 2012). Since in both galaxies the large majority of GCs are uniformly alphaenhanced over a wide range of iron abundance, some clusters with high $[\mathrm{Fe} / \mathrm{H}]$ and high $[\alpha / \mathrm{Fe}]$-ratios should be of accreted origin. In contrast to Ruprecht 106 and G002, these clusters require a progenitor system with a higher stellar mass than Fornax in order to prevent a significant contribution of SNe Ia at these high metallicities. In a $\Lambda$ CDM universe, such high-mass mergers are increasingly unlikely. Consequently, alpha-enhanced accreted GCs necessarily need to originate from only a very small number of disrupted satellite systems. Strikingly, studying the bifurcated age-metallicity relation amongst disc- and halo GCs in the MW, Leaman et al. (2013) come to a very similar conclusion for both the masses and the number of accreted host satellites carrying GC systems. Finally, the case of Fornax shows that not all GCs within a dwarf galaxy need to have the same chemical signature. Therefore GCs with different $[\mathrm{Fe} / \mathrm{H}]$ and $[\alpha / \mathrm{Fe}]$ could have originated in the same system.

It seems, there is another lesson to be learned from the observation of alpha-depleted GCs. There is no evidence, neither from observations nor from theory, about the lower mass limit for a galaxy to be able to form (and hold) own GCs. The search and detection of alpha-depleted clusters can serve as an empirical upper limit. With decreasing $[\mathrm{Fe} / \mathrm{H}]$ observed in these peculiar clusters, the lower becomes the required star formation efficiency - and hence mass - of the host. Currently, this limit is set by G002, the most metal-deficient alpha-depleted cluster with $[\mathrm{Fe} / \mathrm{H}]=-1.66$. This cluster consequently requires a host galaxy even smaller than Fornax, and therefore with a maximum mass of $10^{7} M_{\odot}$.

\subsection{H4: the nucleus of the Fornax dSph?}

$\mathrm{H} 4$ is located remarkably close to the center of the Fornax $\mathrm{dSph}$. This, together with its distinguishing higher metallicity compared to the other clusters, has fired speculations about whether $\mathrm{H} 4$ is in fact the nucleated core of the galaxy, similar to M54 in the Sagittarius dSph galaxy (Hardy 2002; Strader et al. 2003) or comparable to the suspect accreted nucleus $\omega$ Cen (Bekki \& Freeman 2003). If this is the case, it is not self-evident if the properties we observe in $\mathrm{H} 4$ can be transferred to classical GC systems in other galaxies.

First, M54 and specifically $\omega$ Cen display a spread in iron. This characteristic cannot be constrained with our data nor from integrated light. We do not observe sufficient individual stars with sufficient chemical precision in order to place a limit on the intrinsic metallicity spread of the system. Integrated light spectroscopy, on the other hand, only provides a cumulative iron abundance while the necessary information about linestrength variation is lost in the doppler-broadened line profile (McWilliam \& Bernstein 2008).

Second, M54 is embedded in the very center of its host galaxy. There is also no clear answer to this criterion. If $\mathrm{H} 4$ in fact falls on top of the cusp of the field stellar distribution is a matter of debate because of the asymmetry in Fornax' density profile (Stetson et al. 1998). This results in a "chaotic" behaviour of centroids and inclination angles for elliptical profiles fitted at different radii (Demers et al. 1994). While these authors claim to find $\mathrm{H} 4$ at the position where the surface density of stars peaks, Hodge (1961) and later Stetson et al. (1998) find an offset between the peak density and the position of H4. If H4 is the nucleated core of Fornax, it should also be measured at the exact same distance. From ZAHB models we find a best fitting distance modulus of $\mu_{0}=20.74$, which agrees with previous distance measurements for the field star population in Fornax, ranging between $\mu_{0}=20.65$ (Bersier 2000) and 20.87 (Pietrzyński et al. 2003). Given that the actual physical size of the galaxy is between 2 and $3 \mathrm{kpc}$, and by that several times smaller than the uncertainty on its distance estimations, the existing measurements allow for a placement of $\mathrm{H} 4$ right in the center of the galaxy as well as several times outside its tidal perimeter.

Third, M54 moves with the main body of Sagittarius. As outlined in Sect. 4.1, the radial velocity of $\mathrm{H} 4$ is determined as precise as $1 \mathrm{~km} \mathrm{~s}^{-1}$ from integrated-light spectroscopy, and is found to be distinctively different to the mean galactic motion by $\sim 9 \mathrm{~km} \mathrm{~s}^{-1}$. This, finally, is evidence that $\mathrm{H} 4$ is a classical GC which just coincidentally falls close to the line-of-sight towards the center of Fornax. Better data will be necessary to further constrain the first and the second aspects, and to eventually obtain a final conclusion on the nature of $\mathrm{H} 4$.

\subsection{On the discrepancy between photometric and spectroscopic metallicity estimates for $\mathrm{H} 4$}

The literature metallicity estimates of $\mathrm{H} 4$ derived through photometric and spectroscopic methods have yielded inconsistent results: Buonanno et al. (1999) estimates a metallicity $\leq-2.0 \mathrm{dex}$ based on the slope of the RGB as compared to the other Fornax clusters, a result which has been confirmed later by D'Antona et al. (2013). In contrast, spectroscopic measurements consistently yield values around $\sim-1.4$ dex (e.g., Strader et al. 2003; Larsen et al. 2012) - a discrepancy too large to be explained solely by measurement errors. However, while spectroscopic iron-line analyses yield $[\mathrm{Fe} / \mathrm{H}]$, broad-band photometric approaches are sensitive to the total mass fraction of all metals $(Z)$, which determines the stellar structure and hence the cluster fiducial sequence in a CMD. Therefore, it is possible that the low alpha-abundance ratio we find for $\mathrm{H} 4$ is responsible for the discrepancy between photometric and spectroscopic metallicity estimates.

In fact, we find a ratio of metallicity mass fraction between the GCs $\mathrm{H} 4$ and, e.g., $\mathrm{H} 2 Z_{\mathrm{H} 4} / Z_{\mathrm{H} 2} \approx 3.2$, when we assume $[\mathrm{Fe} / \mathrm{H}]=-1.5$ dex for $\mathrm{H} 4$ and -2.1 dex for $\mathrm{H} 2$, and enhanced alpha-abundances (including oxygen) to $[\alpha / \mathrm{Fe}]=+0.4 \mathrm{dex}$ for both clusters. The metallicity mass fraction ratio decreases to only $\approx 1.5$, and hence to about half of the original discrepancy, when we instead adopt solar-like alpha-abundance ratios for $\mathrm{H} 4$. Alternatively, for a cluster with H4's iron abundance, the absolute alteration in $Z$ caused by a change from $[\alpha / \mathrm{Fe}]=+0.4 \mathrm{dex}$ to solar, amounts to $\Delta Z=5 \times 10^{-4}$ when we apply the standard solar abundance scale from Asplund et al. (2009). This number 


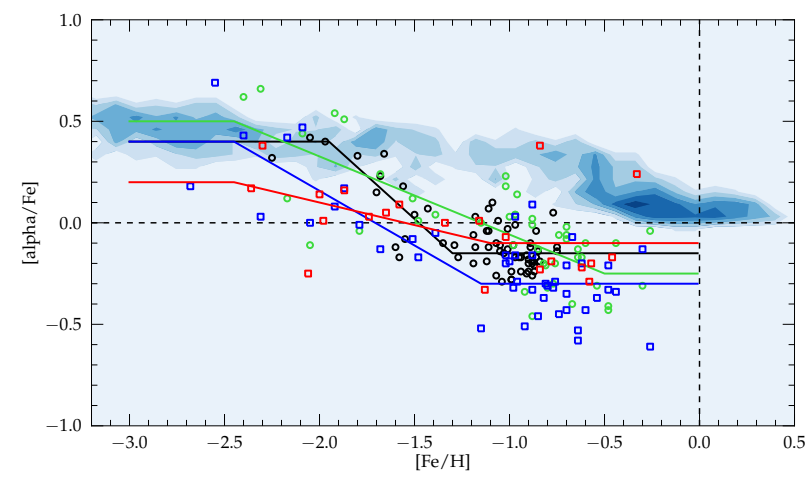

Fig. 12. Fornax displays very similar chemical enrichment signatures at different radial positions within the galaxy. The evolution of individual alpha-elements is identical within the limited precision of the data. In detail, black circles are $[\mathrm{Mg} / \mathrm{Fe}]$ measured at $r \approx 0.6^{\circ}$ from Hendricks et al. (2014a), green circles and blue squares show $[\mathrm{Mg} / \mathrm{Fe}]$ and $[\mathrm{Ca} / \mathrm{Fe}]$ measured at $r \approx 0.6^{\circ}$ from Lemasle et al. (2014), and red squares are the $[\mathrm{Ca} / \mathrm{Fe}]$ values at $r \approx 0.2^{\circ}$ from this work. The lines are toy model fits as described in the text to the samples with corresponding color. The contours show the evolution of MW field stars for comparison, averaged for $\mathrm{Mg}$ and $\mathrm{Ca}$.

is about equivalent to a shift in $[\mathrm{Fe} / \mathrm{H}]$ from $-1.5 \mathrm{dex}$ to $-1.8 \mathrm{dex}$ when $[\alpha / \mathrm{Fe}]$ is kept constant.

Thus, at least part of the discrepancy between photometric and spectroscopic abundance determinations for $\mathrm{H} 4$ likely stems from the low $[\alpha / \mathrm{Fe}]$ ratio in $\mathrm{H} 4$ compared to the other clusters, and from the fact that spectroscopic iron abundances have been compared to features that are more sensitive to the combined mass fraction of elements heavier than helium.

\subsection{Insights from field star evolution at different galactocentric radii}

Fornax is one of the best studied of all dwarf galaxies, and the detailed chemical properties of field stars have been an issue in a series of recent papers (Letarte et al. 2010; Hendricks et al. 2014a; Lemasle et al. 2014). It is also the only galaxy where the combined datasets cover a large fraction of its radial extent. Specifically, Hendricks et al. (2014a) and Lemasle et al. (2014) provide the alpha-evolution of stars at $r \approx 0.6^{\circ}$ (compared to a tidal radius of $\sim 1^{\circ}$ ), while the current work, for the first time, yield similar information for the very central area at $r \leq 0.2^{\circ}$.

In Fig. 12, we fit a simple step function to the alpha-evolution sequence of all literature samples, similarly to what has been done in Cohen \& Huang (2010), or Hendricks et al. (2014a). This toy model has no physical motivation, and is only designed to estimate the position of the knee and the two plateau values of $[\alpha / \mathrm{Fe}]$ for both high and low $[\mathrm{Fe} / \mathrm{H}]$. When we compare the alpha-evolution at different radial positions in this naive way, we find them to follow essentially the same sequence, which also agrees with the visual impression of the data.

This mutual agreement between the different samples confirms a very slow chemical enrichment in Fornax, seen as a metal-poor knee. Moreover, this means that the chemical enrichment efficiency in the center and the outskirts of Fornax had to be similar, at least at early times. This fact is a little surprising, if one considers that the enrichment efficiency comprises the star formation efficiency on the one side, and the ability to retain the processed stellar yields on the other. Both factors are theoretically sensitive to the density of the ISM and the depth of the gravitational potential, which in turn are both a function of radius.
It is well established that stars in the center of Fornax are of a significantly higher average metallicity than in the outer parts (e.g., Battaglia et al. 2006). The consequence of the above considerations could be that the inner area did not undergo a faster chemical evolution, but rather experienced a longer star formation history, which eventually caused the observed radial metallicity gradient within the galaxy.

\section{Summary}

In this paper, we presented radial velocities and high-resolution chemical abundances for $\mathrm{Fe}$, several alpha-elements $(\mathrm{Ca}, \mathrm{Ti}$, and $\mathrm{Si}$ ), and iron-peak elements ( $\mathrm{V}, \mathrm{Cr}, \mathrm{Co}, \mathrm{Ni}$ ) for an individual member star of the peculiar GC H4 and 27 additional stars in the surrounding field at the center of the Fornax dwarf spheroidal galaxy. H4 is difficult to observe due to its central position within the galaxy with severe contamination higher than $30 \%$ in the resolved area of the cluster. Our sample has been selected from HST photometry, where we carefully avoided blended stars in a seeing limited scenario. We obtain the first detailed chemical analysis for an individual member star in $\mathrm{H} 4$, which we put into context to the chemical signatures of field stars in Fornax from this work and from previous studies. The main results are summarized below:

- Our field star sample cover a wide range in metallicity with $-2.3 \leq[\mathrm{Fe} / \mathrm{H}] \leq-0.4$, and show a distinct change in $[\alpha / \mathrm{Fe}]$ as a function of $[\mathrm{Fe} / \mathrm{H}]$. In detail, stars are alpha-enhanced at low metallicity where they show similar properties to metaldeficient MW field stars. With increasing $[\mathrm{Fe} / \mathrm{H}]$, they follow a clear sequence towards sub-solar $[\alpha / \mathrm{Fe}]$ ratios.

- The observed field star sequence is in good agreement with previous observations made in this galaxy and does not allow for a knee in the alpha-evolution significantly higher than $[\mathrm{Fe} / \mathrm{H}] \sim-2.0$ dex, indicative of a low star formation efficiency in Fornax.

- By comparing the alpha-evolution of different field star samples from literature, it is possible to put a tentative constraint on radial chemical enrichment variations within Fornax. We do not find any significant variation for the evolution of a given species with $[\mathrm{Fe} / \mathrm{H}]$ and therefore speculate that such variations, if existent, have to be small.

- We obtain precise chemical abundances for one star with a 99.2\% membership likelihood to the GC H4 and find low alpha-abundances of $[\mathrm{Ca} / \mathrm{Fe}]=+0.05 \pm 0.08$, $[\mathrm{Ti} / \mathrm{Fe}]=$ $-0.27 \pm 0.23$, and $[\mathrm{Si} / \mathrm{Fe}]=-0.35 \pm 0.34$, resulting in an average $[\alpha / \mathrm{Fe}]=-0.19$. This makes $\mathrm{H} 4$ one out of only three metal-deficient GCs known to be alpha-depleted. Moreover, Fornax becomes the first observed birthplace of such peculiar clusters, which can also be found in the halos of larger galaxies, supporting speculations that these clusters are accreted from now disrupted satellite systems.

- Considered together, the GC population in Fornax follows the chemical signature of the field stars and explicitly disagrees with the properties of Milky Way stars and GCs. We therefore conclude that the chemical enrichment of field and clusters in Fornax is coupled and determined by the properties of the common host galaxy.

- If the chemical enrichment signatures of a galaxy as imprinted in the alpha-evolution of its field stars are inherited to its GC population, we can draw inferences from the chemical properties of accreted GCs about their unknown satellite hosts. Following this concept, the alpha-depleted, metal-deficient clusters Ruprecht 106 in the Milky Way and G002 in M31 require a host galaxy similar to, or smaller than 
Fornax, and consequently with a stellar mass of $\sim 10^{7} M_{\odot}$. In contrast, alpha-enhanced GCs with $[\mathrm{Fe} / \mathrm{H}] \sim-1$, if accreted, require more massive birth places with masses $\sim 10^{8} M_{\odot}$ or more. This argument, however, relies on the assumption that mass is the main parameter to determine the chemical enrichment properties of a galaxy. If, however, other parameters like environmental interactions also play an important role, it becomes more difficult to reconstruct the host galaxies properties in such a way.

Finally, it is important to emphasize that a large portion of our findings and their interpretation rely on the properties of only one member star of H4 (two more likely candidates in our sample proved too faint for a detailed chemical analysis). Although the analyzed star is almost certainly a member of the cluster, and despite the robust abundance measurement we could perform on its spectrum, it is possible that its properties are not representative of the average cluster chemical composition. Clearly, in order to confirm the results related to $\mathrm{H} 4$ and the subsequent conclusions, it would be desirable to obtain chemical information from more individual members of this cluster in the future.

Acknowledgements. We thank Charli Sakari, Hans-Günter Ludwig, and Morgan Fouesneau for helpful discussions. The HST data used in this paper were obtained from the Mikulski Archive for Space Telescopes (MAST). STScI is operated by the Association of Universities for Research in Astronomy, Inc., under NASA contract NAS5-26555. This publication makes use of data products from the Two Micron All Sky Survey, which is a joint project of the University of Massachusetts and the Infrared Processing and Analysis Center/California Institute of Technology, funded by NASA and the National ScienceFoundation. B.H., M.F., and A.K. acknowledge the German Research Foundation (DFG) for funding from Emmy-Noether grant Ko 4161/1. This work was in part supported by Sonderforschungsbereich SFB 881 "The Milky Way System" (subproject A5) of the DFG. C.I.J. acknowledges support through the Clay Fellowship administered by the Smithsonian Astrophysical Observatory.

\section{References}

Alonso, A., Arribas, S., \& Martínez-Roger, C. 1999, A\&AS, 140, 261 D'Antona, F., Caloi, V., D'Ercole, A., et al. 2013, MNRAS, 434, 1138 Asplund, M., Grevesse, N., Sauval, A. J., \& Scott, P. 2009, ARA\&A, 47, 481 Battaglia, G., Tolstoy, E., Helmi, A., et al. 2006, A\&A, 459, 423 Battaglia, G., Irwin, M., Tolstoy, E., et al. 2008, MNRAS, 383, 183 Bekki, K., \& Freeman, K. C. 2003, MNRAS, 346, L11 Bensby, T., Feltzing, S., \& Lundström, I. 2003, A\&A, 410, 527 Bersier, D. 2000, ApJ, 543, L23

Boeche, C., \& Grebel, E. 2015, A\&A, in press Boeche, C., Siebert, A., Williams, M., et al. 2011, AJ, 142, 193 de Boer, T. J. L., Tolstoy, E., Hill, V., et al. 2012, A\&A, 544, A73 Brodie, J. P., \& Strader, J. 2006, ARA\&A, 44, 193

Brown, J. A., Wallerstein, G., \& Zucker, D. 1997, AJ, 114, 180

Buonanno, R., Corsi, C. E., Zinn, R., et al. 1998, ApJ, 501, L33

Buonanno, R., Corsi, C. E., Castellani, M., et al. 1999, AJ, 118, 1671

Burgh, E. B., Bershady, M. A., Westfall, K. B., \& Nordsieck, K. H. 2007, PASP, 119,1069

Caffau, E., Koch, A., Sbordone, L., et al. 2013, Astron. Nachr., 334, 197

Carretta, E., Bragaglia, A., Gratton, R. G., et al. 2010, A\&A, 520, A95

Choi, J., Conroy, C., Moustakas, J., et al. 2014, ApJ, 792, 95

Coelho, P., Barbuy, B., Meléndez, J., Schiavon, R. P., \& Castilho, B. V. 2005, A\&A, 443, 735

Cohen, J. G. 2004, AJ, 127, 1545

Cohen, J. G., \& Huang, W. 2010, ApJ, 719, 931

Colucci, J. E., Bernstein, R. A., \& Cohen, J. G. 2014, ApJ, 797, 116

Conroy, C., Graves, G. J., \& van Dokkum, P. G. 2014, ApJ, 780, 33
Cutri, R. M., Skrutskie, M. F., van Dyk, S., et al. 2003, VizieR Online Data Catalog: II/246

Demers, S., Irwin, M. J., \& Kunkel, W. E. 1994, AJ, 108, 1648

van Dokkum, P. G. 2001, PASP, 113, 1420

Dolphin, A. E. 2000, PASP, 112, 1383

Dolphin, A. E. 2009, PASP, 121, 655

Dotter, A., Chaboyer, B., Jevremović, D., et al. 2007, AJ, 134, 376

Dotter, A., Chaboyer, B., Jevremović, D., et al. 2008, ApJS, 178, 89

Dubath, P., Meylan, G., \& Mayor, M. 1992, ApJ, 400, 510

Edge, A., Sutherland, W., Kuijken, K., et al. 2013, The Messenger, 154, 32

Elmegreen, B. G., Malhotra, S., \& Rhoads, J. 2012, ApJ, 757, 9

Frank, M. J., Hilker, M., Baumgardt, H., et al. 2012, MNRAS, 423, 2917

Gratton, R. G., Carretta, E., \& Bragaglia, A. 2012, A\&ARv, 20, 50

Greco, C., Clementini, G., Catelan, M., et al. 2007, A\&ARv, 670, 332

Hardy, E. 2002, Extragalactic Star Clusters, 207, 62

Hendricks, B., Stetson, P. B., VandenBerg, D. A., \& Dall'Ora, M. 2012, AJ, 144, 25

Hendricks, B., Koch, A., Lanfranchi, G. A., et al. 2014a, ApJ, 785, 102

Hendricks, B., Koch, A., Walker, M., et al. 2014b, A\&A, 572, A82

Hodge, P. W. 1961, AJ, 66, 83

Huxor, A. P., Ferguson, A. M. N., Tanvir, N. R., et al. 2011, MNRAS, 414, 770

Johnson, C. I., McDonald, I., Pilachowski, C. A., et al. 2015, AJ, 149, 71

King, I. R. 1966, AJ, 71, 64

Kirby, E. N., Guhathakurta, P., \& Sneden, C. 2008, ApJ, 682, 1217

Koekemoer, A. M., Fruchter, A. S., Hook, R. N., Hack, W., \& Hanley, C. 2006, The 2005 HST Calibration Workshop: Hubble After the Transition to TwoGyro Mode, 423

Lanfranchi, G. A., \& Matteucci, F. 2003, MNRAS, 345, 71

Larsen, S. S., Brodie, J. P., \& Strader, J. 2012, A\&A, 546, A53

Law, D. R., \& Majewski, S. R. 2010, ApJ, 718, 1128

Leaman, R., Venn, K. A., Brooks, A. M., et al. 2013, ApJ, 767, 131

Lemasle, B., de Boer, T. J. L., Hill, V., et al. 2014, A\&A, 572, A88

Letarte, B., Hill, V., Jablonka, P., et al. 2006, A\&A, 453, 547

Letarte, B., Hill, V., Tolstoy, E., et al. 2010, A\&A, 523, A17

Mackey, A. D., \& Gilmore, G. F. 2003, MNRAS, 340, 175

Mackey, A. D., \& Gilmore, G. F. 2004, MNRAS, 355, 504

Mackey, A. D., Huxor, A. P., Ferguson, A. M. N., et al. 2010, ApJ, 717, L11

Mateo, M., Bailey, J. I., Crane, J., et al. 2012, Proc. SPIE, 8446, 84464

Matteucci, F., \& Brocato, E. 1990, ApJ, 365, 539

McConnachie, A. W. 2012, AJ, 144, 4

McWilliam, A., \& Bernstein, R. A. 2008, ApJ, 684, 326

Pietrzyński, G., Gieren, W., \& Udalski, A. 2003, AJ, 125, 2494

Pietrzyński, G., Górski, M., Gieren, W., et al. 2009, AJ, 138, 459

del Pino, A., Hidalgo, S. L., Aparicio, A., et al. 2013, MNRAS, 433, 1505

Pompéia, L., Hill, V., Spite, M., et al. 2008, A\&A, 480, 379

Pritzl, B. J., Venn, K. A., \& Irwin, M. 2005, AJ, 130, 2140

Reddy, B. E., Tomkin, J., Lambert, D. L., \& Allende Prieto, C. 2003, MNRAS, 340,304

Rizzi, L., Held, E. V., Saviane, I., Tully, R. B., \& Gullieuszik, M. 2007, MNRAS, 380, 1255

Roederer, I. U., Preston, G. W., Thompson, I. B., et al. 2014, AJ, 147, 136

Sakari, C. M., Venn, K. A., Mackey, D., et al. 2015, MNRAS, 448, 1314

Sbordone, L., Bonifacio, P., Marconi, G., Buonanno, R., \& Zaggia, S. 2005, A\&A, 437, 905

Sbordone, L., Bonifacio, P., Buonanno, R., et al. 2007, A\&A, 465, 815

Schlegel, D. J., Finkbeiner, D. P., \& Davis, M. 1998, ApJ, 500, 525

Stetson, P. B., Hesser, J. E., \& Smecker-Hane, T. A. 1998, PASP, 110, 533

Stetson, P. B., Bruntt, H., \& Grundahl, F. 2003, PASP, 115, 413

Strader, J., Brodie, J. P., Forbes, D. A., Beasley, M. A., \& Huchra, J. P. 2003, AJ, 125,1291

Tafelmeyer, M., Jablonka, P., Hill, V., et al. 2010, A\&A, 524, A58

Tinsley, B. M. 1979, ApJ, 229, 1046

Tolstoy, E., Hill, V., \& Tosi, M. 2009, ARA\&A, 47, 371

VandenBerg, D. A., Brogaard, K., Leaman, R., \& Casagrande, L. 2013, ApJ, 775,134

Venn, K. A., Irwin, M., Shetrone, M. D., et al. 2004, AJ, 128, 1177

Villanova, S., Geisler, D., Carraro, G., Moni Bidin, C., \& Muñoz, C. 2013, ApJ, 778, 186 
B. Hendricks et al.: Chemical coupling of GCs and field stars in the Fornax dSph

Appendix A: Abundances and velocities for Fornax field stars and likely members of H4

Table A.1. Basic parameters - positions, photometry, and quality of the spectra.

\begin{tabular}{|c|c|c|c|c|c|c|c|c|c|c|}
\hline Star ID & $\alpha$ & $\delta$ & Group & V & $\sigma V$ & $V-I$ & $\sigma(V-I)$ & $m_{\mathrm{sep}}$ & $S / N$ & $\Delta \lambda_{\mathrm{eff}}[\AA]$ \\
\hline r_0006 & $2 \mathrm{~h} 40 \mathrm{~m} 10.15 \mathrm{~s}$ & $-34 \mathrm{~d} 31 \mathrm{~m} 48.83 \mathrm{~s}$ & A & 18.892 & 0.007 & 1.260 & 0.009 & 10.01 & 23.8 & 301.6 \\
\hline r_0007 & $2 \mathrm{~h} 40 \mathrm{~m} 9.19 \mathrm{~s}$ & $-34 \mathrm{~d} 32 \mathrm{~m} 59.89 \mathrm{~s}$ & A & 19.540 & 0.010 & 1.116 & 0.013 & 6.87 & 15.9 & 393.9 \\
\hline r_0008 & $2 \mathrm{~h} 40 \mathrm{~m} 5.51 \mathrm{~s}$ & $-34 \mathrm{~d} 32 \mathrm{~m} 42.86 \mathrm{~s}$ & A & 18.326 & 0.005 & 1.586 & 0.006 & 8.16 & 40.2 & 439.2 \\
\hline r_0009 & $2 \mathrm{~h} 40 \mathrm{~m} 8.84 \mathrm{~s}$ & $-34 \mathrm{~d} 32 \mathrm{~m} 47.83 \mathrm{~s}$ & A & 19.249 & 0.008 & 1.196 & 0.011 & 6.82 & 18.0 & 214.2 \\
\hline r_0010* & $2 \mathrm{~h} 40 \mathrm{~m} 7.69 \mathrm{~s}$ & $-34 \mathrm{~d} 32 \mathrm{~m} 0.92 \mathrm{~s}$ & A & 18.805 & 0.007 & 1.231 & 0.009 & 5.95 & 33.7 & 345.7 \\
\hline r_0011 & $2 \mathrm{~h} 40 \mathrm{~m} 8.30 \mathrm{~s}$ & $-34 \mathrm{~d} 32 \mathrm{~m} 37.28 \mathrm{~s}$ & A & 18.818 & 0.007 & 1.327 & 0.009 & 8.49 & 27.4 & 450.6 \\
\hline r_0014 & $2 \mathrm{~h} 39 \mathrm{~m} 41.20 \mathrm{~s}$ & $-34 \mathrm{~d} 34 \mathrm{~m} 50.09 \mathrm{~s}$ & B & 18.242 & - & 1.260 & - & - & 33.4 & 434.7 \\
\hline r_0016 & $2 \mathrm{~h} 40 \mathrm{~m} 6.63 \mathrm{~s}$ & $-34 \mathrm{~d} 32 \mathrm{~m} 25.80 \mathrm{~s}$ & A & 20.068 & 0.004 & 1.041 & 0.005 & 4.70 & 13.8 & 175.3 \\
\hline r_0017 & $2 \mathrm{~h} 40 \mathrm{~m} 7.22 \mathrm{~s}$ & $-34 \mathrm{~d} 31 \mathrm{~m} 44.94 \mathrm{~s}$ & A & 19.488 & 0.003 & 1.174 & 0.004 & 4.75 & 17.3 & 304.7 \\
\hline r_0018 & $2 \mathrm{~h} 40 \mathrm{~m} 7.07 \mathrm{~s}$ & $-34 \mathrm{~d} 31 \mathrm{~m} 25.79 \mathrm{~s}$ & $\mathrm{~A}$ & 19.196 & 0.008 & 1.142 & 0.011 & 4.73 & 19.4 & 379.4 \\
\hline r_0019 & $2 \mathrm{~h} 39 \mathrm{~m} 31.50 \mathrm{~s}$ & $-34 \mathrm{~d} 46 \mathrm{~m} 45.12 \mathrm{~s}$ & B & 18.082 & - & 1.020 & - & - & 34.4 & 423.2 \\
\hline r_0020 & $2 \mathrm{~h} 39 \mathrm{~m} 4.80 \mathrm{~s}$ & $-34 \mathrm{~d} 41 \mathrm{~m} 31.49 \mathrm{~s}$ & B & 18.422 & - & 1.230 & - & - & 43.8 & 463.3 \\
\hline r_0021 & $2 \mathrm{~h} 39 \mathrm{~m} 12.29 \mathrm{~s}$ & $-34 \mathrm{~d} 44 \mathrm{~m} 38.54 \mathrm{~s}$ & B & 17.912 & - & 1.240 & - & - & 39.5 & 478.8 \\
\hline r_0022 & $2 \mathrm{~h} 40 \mathrm{~m} 4.01 \mathrm{~s}$ & $-34 \mathrm{~d} 32 \mathrm{~m} 13.60 \mathrm{~s}$ & A & 19.312 & 0.009 & 1.144 & 0.012 & 8.84 & 21.3 & 378.0 \\
\hline r_0024 & $2 \mathrm{~h} 40 \mathrm{~m} 4.46 \mathrm{~s}$ & $-34 \mathrm{~d} 31 \mathrm{~m} 38.64 \mathrm{~s}$ & A & 19.532 & 0.010 & 1.161 & 0.013 & 5.62 & 18.5 & 360.6 \\
\hline b_0001 & $2 \mathrm{~h} 39 \mathrm{~m} 11.42 \mathrm{~s}$ & $-34 \mathrm{~d} 29 \mathrm{~m} 22.27 \mathrm{~s}$ & B & 18.012 & - & 1.320 & - & - & 16.6 & 277.0 \\
\hline b_0003 & $2 \mathrm{~h} 39 \mathrm{~m} 39.90 \mathrm{~s}$ & $-34 \mathrm{~d} 43 \mathrm{~m} 7.21 \mathrm{~s}$ & B & 18.362 & - & 1.110 & - & - & 51.1 & 375.9 \\
\hline b_0005 & $2 \mathrm{~h} 39 \mathrm{~m} 36.30 \mathrm{~s}$ & $-34 d 51 m 25.99 s$ & B & 18.062 & - & 1.710 & - & - & 19.5 & 379.4 \\
\hline b_0007 & $2 \mathrm{~h} 40 \mathrm{~m} 5.97 \mathrm{~s}$ & $-34 \mathrm{~d} 32 \mathrm{~m} 0.89 \mathrm{~s}$ & A & 18.367 & 0.006 & 1.397 & 0.007 & 7.56 & 38.1 & 403.0 \\
\hline b_0008 & $2 \mathrm{~h} 40 \mathrm{~m} 6.09 \mathrm{~s}$ & $-34 \mathrm{~d} 31 \mathrm{~m} 38.28 \mathrm{~s}$ & A & 18.883 & 0.007 & 1.236 & 0.009 & 4.22 & 20.3 & 311.7 \\
\hline b_0009 & $2 \mathrm{~h} 39 \mathrm{~m} 55.38 \mathrm{~s}$ & $-34 \mathrm{~d} 45 \mathrm{~m} 56.05 \mathrm{~s}$ & B & 18.282 & - & 1.480 & - & - & 26.9 & 355.3 \\
\hline b_0010 & $2 \mathrm{~h} 39 \mathrm{~m} 53.79 \mathrm{~s}$ & $-34 \mathrm{~d} 38 \mathrm{~m} 55.50 \mathrm{~s}$ & B & 18.322 & - & 1.160 & - & - & 29.4 & 175.3 \\
\hline b_0011 & $2 \mathrm{~h} 39 \mathrm{~m} 52.62 \mathrm{~s}$ & $-34 \mathrm{~d} 45 \mathrm{~m} 44.60 \mathrm{~s}$ & B & 17.982 & - & 1.290 & - & - & 55.5 & 426.4 \\
\hline b_0015 & $2 \mathrm{~h} 40 \mathrm{~m} 5.09 \mathrm{~s}$ & $-34 \mathrm{~d} 35 \mathrm{~m} 41.75 \mathrm{~s}$ & B & 18.332 & - & 1.160 & - & - & 51.6 & 281.2 \\
\hline b_0016 & $2 \mathrm{~h} 40 \mathrm{~m} 2.73 \mathrm{~s}$ & $-34 \mathrm{~d} 38 \mathrm{~m} 30.05 \mathrm{~s}$ & B & 18.162 & - & 1.260 & - & - & 33.8 & 352.0 \\
\hline b_0017 & $2 \mathrm{~h} 39 \mathrm{~m} 57.10 \mathrm{~s}$ & $-34 \mathrm{~d} 49 \mathrm{~m} 7.03 \mathrm{~s}$ & B & 18.412 & - & 1.080 & - & - & 27.8 & 372.3 \\
\hline b_0018 & $2 \mathrm{~h} 40 \mathrm{~m} 10.92 \mathrm{~s}$ & $-34 \mathrm{~d} 32 \mathrm{~m} 4.31 \mathrm{~s}$ & A & 19.913 & 0.004 & 1.053 & 0.005 & 6.50 & 11.1 & 277.7 \\
\hline b_0019 & $2 \mathrm{~h} 40 \mathrm{~m} 11.20 \mathrm{~s}$ & $-34 \mathrm{~d} 31 \mathrm{~m} 46.78 \mathrm{~s}$ & $\mathrm{~A}$ & 19.627 & 0.010 & 1.143 & 0.013 & 7.20 & 11.1 & 236.1 \\
\hline b_0020 & $2 \mathrm{~h} 40 \mathrm{~m} 21.48 \mathrm{~s}$ & $-34 \mathrm{~d} 25 \mathrm{~m} 57.43 \mathrm{~s}$ & B & 18.402 & - & 1.320 & - & - & 13.2 & 175.0 \\
\hline b_0023 & $2 \mathrm{~h} 40 \mathrm{~m} 30.22 \mathrm{~s}$ & $-34 \mathrm{~d} 38 \mathrm{~m} 53.81 \mathrm{~s}$ & B & 18.142 & - & 1.210 & - & - & 37.1 & 447.7 \\
\hline
\end{tabular}

Notes. "Group" indicates whether the star has been selected within the tidal radius of H4 (A), or whether it is a field star from the central part of Fornax (B). For stars of category A, the seperation index $m_{\text {sep }}$ is an indicator for the flux contamination of a spectrum, as described in Sect. 2.2.1 of the main article. The $\mathrm{S} / \mathrm{N}$ is per pixel of the degraded spectra as they are supplied to SP_AcE. The effective wavelength range $\Delta \lambda_{\text {eff }}$ gives the final wavelength range which is used in the chemical analysis after critical regions flagged in various pixel masks have been removed. ${ }^{(*)}$ The star with ID r_0010 is the target with $99.2 \%$ H4 membership probability. 
Table A.2. Chemical abundances $-[\mathrm{Fe} / \mathrm{H}]$, and alpha-elements.

\begin{tabular}{|c|c|c|c|c|c|c|c|c|c|c|c|}
\hline Star ID & $v$ & $\sigma v$ & {$[\mathrm{Fe} / \mathrm{H}]$} & {$[\mathrm{Fe} / \mathrm{H}]_{+}$} & {$[\mathrm{Fe} / \mathrm{H}]_{-}$} & {$[\mathrm{Ca} / \mathrm{H}]$} & {$[\mathrm{Ca} / \mathrm{H}]_{+}$} & {$[\mathrm{Ca} / \mathrm{H}]_{-}$} & {$[\mathrm{Ti} / \mathrm{H}]$} & {$[\mathrm{Ti} / \mathrm{H}]_{+}$} & {$[\mathrm{Ti} / \mathrm{H}]_{-}$} \\
\hline r_0006 & 53.26 & 0.50 & -1.71 & -1.83 & -1.56 & - & - & - & - & - & - \\
\hline r_0007 & 70.38 & 0.61 & -1.16 & -1.26 & -1.11 & -1.15 & -1.26 & -1.02 & -0.73 & -0.87 & -0.53 \\
\hline r_0008 & 72.10 & 0.20 & -0.62 & -0.72 & -0.54 & -0.84 & -0.99 & -0.66 & -1.01 & -1.10 & -0.77 \\
\hline r_0009 & 58.10 & 0.40 & -0.84 & -1.04 & -0.78 & -0.46 & -0.65 & -0.22 & -1.06 & - & -0.78 \\
\hline r_0010* & 48.16 & 0.36 & -1.50 & -1.54 & -1.45 & -1.44 & -1.53 & -1.37 & -1.77 & - & -1.54 \\
\hline r_0011 & 49.89 & 0.28 & -0.78 & -0.89 & -0.77 & -0.97 & -1.03 & -0.88 & -0.98 & -1.02 & -0.88 \\
\hline r_0014 & 48.94 & 0.25 & -1.13 & -1.18 & -1.11 & -1.46 & -1.51 & -1.38 & -1.38 & -1.49 & -1.32 \\
\hline r_0016 & 46.56 & 0.82 & -1.68 & -1.95 & -1.55 & - & - & - & - & - & - \\
\hline r_0017 & 47.17 & 2.12 & -0.46 & -0.54 & -0.19 & -0.63 & -0.84 & -0.47 & - & - & - \\
\hline r_0018 & 72.78 & 1.83 & -2.30 & - & -2.25 & -1.92 & -2.15 & -1.67 & - & - & - \\
\hline r_0019 & 64.60 & 0.34 & -1.34 & -1.61 & -1.29 & -1.34 & -1.67 & -1.15 & -1.59 & - & -1.21 \\
\hline r_0020 & 58.98 & 1.44 & -1.58 & -1.65 & -1.55 & -1.49 & -1.57 & -1.43 & -1.60 & -1.76 & -1.51 \\
\hline r_0021 & 65.38 & 0.27 & -1.74 & -1.81 & -1.72 & -1.71 & -1.79 & -1.64 & -1.70 & -1.85 & -1.64 \\
\hline r 0022 & 55.72 & 0.28 & -0.84 & -0.99 & -0.82 & -1.07 & -1.16 & -0.95 & -0.96 & -1.12 & -0.79 \\
\hline r_0024 & 61.77 & 0.50 & -0.58 & -0.70 & -0.55 & -0.87 & -1.02 & -0.72 & -1.20 & - & -1.01 \\
\hline b_0001 & 74.85 & 1.07 & -1.40 & -1.50 & -1.30 & -1.94 & -2.05 & -1.69 & -1.61 & -1.81 & -1.26 \\
\hline b_0003 & 67.45 & 0.48 & -2.36 & -2.72 & -2.28 & -2.19 & -2.61 & -1.96 & -1.77 & - & - \\
\hline b_0005 & 39.52 & 0.48 & -0.82 & - & - & - & - & - & - & - & - \\
\hline b_0007 & 50.57 & 0.19 & -1.02 & -1.11 & -0.99 & -1.09 & -1.18 & -0.94 & -1.49 & -1.61 & -1.31 \\
\hline b_0008 & 45.67 & 0.44 & -0.57 & -0.72 & -0.55 & -0.77 & -0.85 & -0.65 & - & - & - \\
\hline b_0009 & 51.13 & 0.33 & -1.34 & - & - & - & - & - & -1.06 & - & - \\
\hline b_0010 & 44.28 & 0.56 & -2.00 & -2.19 & -1.88 & -1.86 & -2.11 & -1.49 & -2.18 & - & -1.68 \\
\hline b_0011 & 47.57 & 0.22 & -2.06 & -2.08 & -1.91 & -2.31 & -2.38 & -2.17 & -1.98 & -2.18 & -1.79 \\
\hline b_0015 & 45.82 & 0.26 & -1.87 & -1.96 & -1.86 & -1.71 & -1.77 & -1.68 & -1.62 & -1.70 & -1.55 \\
\hline b_0016 & 69.05 & 0.24 & -1.65 & -1.72 & -1.63 & -1.60 & -1.71 & -1.56 & -1.64 & -1.73 & -1.49 \\
\hline b_0017 & 70.52 & 0.63 & -1.98 & -2.36 & -1.81 & -1.97 & - & -1.76 & - & - & - \\
\hline b_0018 & 47.18 & 0.77 & -1.46 & - & -0.90 & - & - & - & - & - & - \\
\hline b_0019 & 60.68 & 1.16 & -0.21 & -0.37 & - & -1.20 & - & -0.20 & -0.88 & - & -0.36 \\
\hline b_0020 & 67.04 & 0.66 & -1.65 & -2.01 & -1.57 & -1.84 & - & -1.57 & - & - & - \\
\hline b_0023 & 57.07 & 0.15 & -0.33 & -0.42 & -0.22 & -0.09 & -0.34 & 0.08 & -0.10 & -0.37 & -0.01 \\
\hline
\end{tabular}

Notes. ${ }^{(*)}$ The star with ID r_0010 is the target with $99.2 \%$ H4 membership probability. 
B. Hendricks et al.: Chemical coupling of GCs and field stars in the Fornax dSph

Table A.3. Chemical abundances - iron-peak elements.

\begin{tabular}{|c|c|c|c|c|c|c|c|c|c|c|c|c|}
\hline Star ID & {$[\mathrm{V} / \mathrm{H}]$} & {$[\mathrm{V} / \mathrm{H}]_{+}$} & {$[\mathrm{V} / \mathrm{H}]_{-}$} & {$[\mathrm{Cr} / \mathrm{H}]$} & {$[\mathrm{Cr} / \mathrm{H}]_{+}$} & {$[\mathrm{Cr} / \mathrm{H}]_{-}$} & {$[\mathrm{Co} / \mathrm{H}]$} & {$[\mathrm{Co} / \mathrm{H}]_{+}$} & {$[\mathrm{Co} / \mathrm{H}]_{-}$} & {$[\mathrm{Ni} / \mathrm{H}]$} & {$[\mathrm{Ni} / \mathrm{H}]_{+}$} & {$[\mathrm{Ni} / \mathrm{H}]_{-}$} \\
\hline r_0006 & -1.54 & -2.00 & -1.33 & -1.56 & - & -1.04 & -1.09 & -1.33 & - & -1.79 & - & -1.33 \\
\hline r_0007 & -0.91 & -1.01 & -0.70 & - & - & - & -0.94 & -1.10 & -0.73 & -1.26 & -1.36 & -1.11 \\
\hline r_0008 & -0.92 & -1.00 & -0.68 & -0.95 & -1.16 & -0.61 & -0.96 & -1.10 & -0.73 & -1.00 & -1.19 & -0.83 \\
\hline r_0009 & -0.25 & -0.58 & - & -0.50 & -0.78 & -0.14 & -1.06 & - & -0.82 & -0.55 & -0.86 & -0.36 \\
\hline r_0010* & -1.65 & -1.73 & -1.49 & -1.47 & -1.64 & -1.25 & -1.83 & -1.95 & -1.70 & -1.59 & -1.67 & -1.49 \\
\hline r_0011 & -1.12 & -1.16 & -0.99 & -0.81 & -0.92 & -0.70 & -1.04 & -1.13 & -0.94 & -1.16 & -1.26 & -1.11 \\
\hline r_0014 & -1.41 & -1.49 & -1.32 & -1.64 & - & -1.47 & -1.21 & -1.29 & -1.13 & -1.22 & -1.29 & -1.17 \\
\hline r_0016 & - & - & - & - & - & - & -1.21 & -1.68 & -0.89 & -1.44 & -1.84 & -1.11 \\
\hline r_0017 & -0.76 & - & -0.58 & -0.62 & - & -0.31 & -0.53 & -0.77 & -0.27 & -0.75 & -0.91 & -0.54 \\
\hline r_0018 & - & - & - & - & - & - & -1.65 & -2.48 & - & -2.30 & - & -2.01 \\
\hline r_0019 & -1.91 & - & -1.38 & - & - & - & -1.82 & - & -1.25 & -1.69 & - & -1.43 \\
\hline r_0020 & -2.15 & - & -2.05 & -1.58 & -1.83 & -1.28 & -2.01 & - & -1.85 & -1.84 & -1.97 & -1.76 \\
\hline r_0021 & -2.03 & -2.18 & -1.96 & -2.31 & - & -1.97 & -1.95 & -2.14 & -1.81 & -2.15 & -2.31 & -2.07 \\
\hline r_0022 & -1.11 & -1.17 & -0.85 & -0.97 & -1.21 & -0.68 & -0.96 & -1.11 & -0.79 & -1.10 & -1.21 & -1.00 \\
\hline r_0024 & -0.86 & -0.97 & -0.64 & -0.63 & -0.90 & -0.35 & -0.79 & -1.11 & -0.54 & -1.00 & -1.15 & -0.91 \\
\hline b_0001 & -1.99 & -2.11 & -1.66 & - & - & - & -1.62 & -1.93 & -1.30 & - & - & - \\
\hline b_0003 & -2.23 & - & - & - & - & - & -2.80 & - & -1.77 & -2.85 & - & -2.45 \\
\hline b_0005 & -0.92 & - & - & -1.12 & - & - & - & - & - & -1.03 & - & - \\
\hline b_0007 & -1.55 & -1.62 & -1.34 & -1.51 & -1.70 & -1.25 & -1.21 & -1.38 & -1.08 & -1.35 & -1.49 & -1.26 \\
\hline b_0008 & -0.91 & -1.00 & -0.69 & -0.92 & -1.16 & -0.67 & -0.96 & -1.09 & -0.83 & -0.91 & -1.06 & -0.83 \\
\hline b_0009 & -1.04 & - & - & -1.37 & - & - & -1.34 & - & - & - & - & - \\
\hline b_0010 & - & - & - & - & - & - & -1.49 & -1.97 & - & -2.16 & - & -1.84 \\
\hline b_0011 & -2.65 & - & -2.37 & - & - & - & - & - & - & -2.58 & - & -2.41 \\
\hline b_0015 & - & - & - & - & - & - & -2.20 & - & -2.07 & -1.70 & -1.77 & -1.65 \\
\hline b_0016 & -1.83 & -2.00 & -1.78 & - & - & - & -1.99 & - & -1.91 & -1.68 & -1.81 & -1.62 \\
\hline b_0017 & - & - & - & - & - & - & -1.76 & - & - & -2.16 & - & - \\
\hline b_0018 & - & - & - & - & - & - & - & - & - & - & - & - \\
\hline b_0019 & - & - & - & - & - & - & - & - & - & -0.99 & - & -0.51 \\
\hline b_0020 & - & - & - & - & - & - & - & - & - & - & - & - \\
\hline b_0023 & 0.11 & -0.19 & 0.20 & -0.20 & -0.58 & 0.01 & -0.35 & -0.62 & -0.13 & -0.56 & - & -0.34 \\
\hline
\end{tabular}

Notes. ${ }^{(*)}$ The star with ID r_0010 is the target with $99.2 \%$ H4 membership probability. 